\title{
Self-Avoiding Walk in Five or More Dimensions I. The Critical Behaviour
}

\author{
Takashi Hara ${ }^{1 \star}$ and Gordon Slade ${ }^{2 \star \star}$ \\ ${ }^{1}$ Department of Physics, Gakushuin University, Toshima-ku, Tokyo 171, Japan \\ 2 Department of Mathematics and Statistics, McMaster University, Hamilton, Ontario, \\ Canada L8S 4K1
}

Received May 1, 1991; in revised form November 27, 1991

\begin{abstract}
We use the lace expansion to study the standard self-avoiding walk in the $d$-dimensional hypercubic lattice, for $d \geqq 5$. We prove that the number $c_{n}$ of $n$-step self-avoiding walks satisfies $c_{n} \sim A \mu^{n}$, where $\mu$ is the connective constant (i.e. $\gamma=1$ ), and that the mean square displacement is asymptotically linear in the number of steps (i.e. $v=1 / 2$ ). A bound is obtained for $c_{n}(x)$, the number of $n$-step self-avoiding walks ending at $x$. The correlation length is shown to diverge asymptotically like $\left(\mu^{-1}-z\right)^{-1 / 2}$. The critical two-point function is shown to decay at least as fast as $|x|^{-2}$, and its Fourier transform is shown to be asymptotic to a multiple of $k^{-2}$ as $k \rightarrow 0$ (i.e. $\eta=0$ ). We also prove that the scaling limit is Gaussian, in the sense of convergence in distribution to Brownian motion. The infinite self-avoiding walk is constructed. In this paper we prove these results assuming convergence of the lace expansion. The convergence of the lace expansion is proved in a companion paper.
\end{abstract}

\section{Contents}

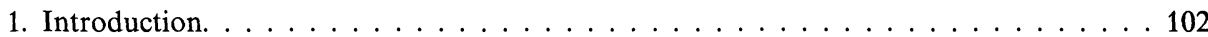

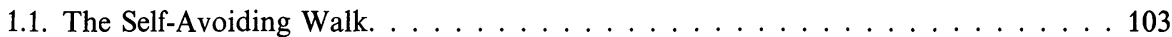

1.2. Main Results. . . . . . . . . . . . . . . . . . . . . . . . . . 104

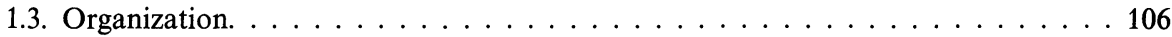

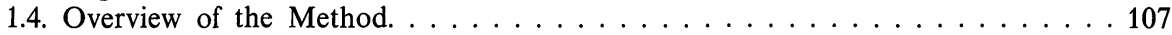

2. The Lace Expansion. . . . . . . . . . . . . . . . . . . . . . . . . 109

2.1. General Description. . . . . . . . . . . . . . . . . . . . . . . . . 109

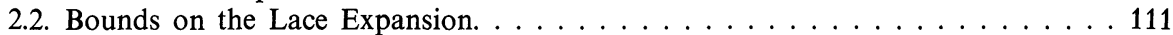

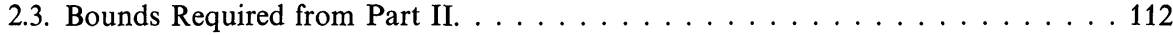

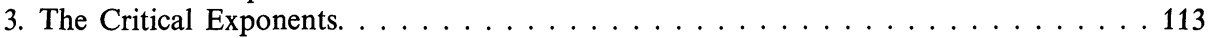

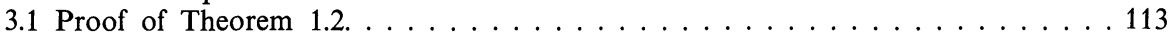

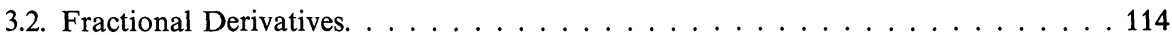

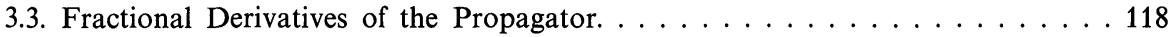

ऋ Supported by the Nishina Memorial Foundation and NSF grant PHY-8896163. Present address: Department of Applied Physics, Tokyo Institute of Technology, Oh-Okayama, Meguroku, Tokyo 152, Japan, electronic address: hara@appana. ap. titech. ac.jp

$\star \star$ Supported by NSERC grant A9351, electronic address: slade@aurora.math.mcmaster.ca 
3.4. Proof of Theorem 1.1. . . . . . . . . . . . . . . . . . . . . . . . 122

3.5. Proof of Theorem 1.4 . . . . . . . . . . . . . . . . . . . . . 124

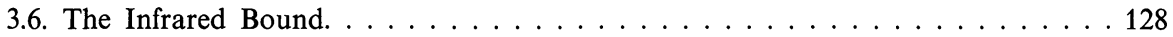

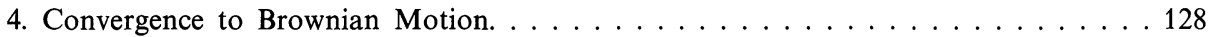

4.1. The Scaling Limit of the Endpoint. . . . . . . . . . . . . . . . . . . . . . 129

4.2. The Finite-Dimensional Distributions. . . . . . . . . . . . . . . 131

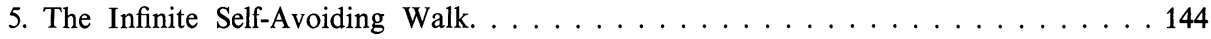

\section{Introduction}

The self-avoiding walk is a simply defined mathematical model with important applications in polymer chemistry and statistical physics. It also serves as a basic example of a non-Markovian process, but lies beyond the reach of current methods of probability theory. In addition, it poses simply stated combinatorial questions which have not yet met a mathematically satisfactory resolution.

It has been conjectured for some time that the self-avoiding walk behaves like simple random walk in more than four dimensions. In four dimensions logarithmic corrections to the scaling behaviour of the self-avoiding walk are expected, while below four dimensions quite different behaviour is expected. This "triviality" of the self-avoiding walk above four dimensions is analogous to the triviality of $\varphi^{4}$ field theory in more than four dimensions $[1,10]$, and more generally to the existence of an upper critical dimension for statistical mechanical models. The proof of triviality of the $\varphi^{4}$ theory relies on the infrared bound, for which there is no general proof known for the self-avoiding walk, and does not extend readily to the self-avoiding walk.

Brydges and Spencer [8] proved Gaussian behaviour for the weakly selfavoiding walk (or Domb-Joyce model) above four dimensions, if the interaction is sufficiently weak, using the lace expansion. This provided strong evidence that the standard self-avoiding walk is also Gaussian above four dimensions, according to the conjecture that the weakly self-avoiding walk is in the same universality class as the standard self-avoiding walk. The lace expansion was subsequently applied to study the standard self-avoiding walk above some high undetermined dimension $d_{0}$ $[32-34,24]$. The lace expansion has proved to be a versatile tool, and has been applied successfully to percolation $[16,15]$, oriented percolation [29], and branched polymers $[17,19]$.

In this and a companion paper [20] we extend and improve the results for the self-avoiding walk in dimensions $d \geqq d_{0}$ to $d \geqq 5$, using the lace expansion. The small parameter responsible for convergence of the lace expansion is the "bubble diagram," whose Gaussian value for $d=5$ is $\sum_{x \neq 0} C(x)^{2}=0.5979$, where $C(x)=\sum_{\omega: 0 \rightarrow x}(1 / 2 d)^{|\omega|}$, the sum being over all simple random walks from 0 to $x$. Because this value is so large, convergence of the expansion is a much more significant problem here than in the previous results for which the small parameter could be taken arbitrarily small. Computer calculations (with controlled errors) have been used to perform some of the calculations involved in the convergence proof. As a result good numerical bounds have been obtained, e.g. for the diffusion constant. The proof of convergence of the expansion is treated in the companion paper [20], which we shall refer to as Part II.

The previous methods used to study the self-avoiding walk for $d \geqq d_{0}$ could not be extended directly to $d \geqq 5$, due to the use of derivatives of Green's functions which diverge for $d=5$. To overcome this we have developed a method using 
convergent fractional derivatives of Green's functions instead. (Fractional derivatives were also used by Brydges and Spencer [8], although not referred to by that name and with a quite different approach.)

Our method also differs from previous applications of the lace expansion to the self-avoiding walk, in that we work directly with the self-avoiding walk and need not consider finite-memory walks, which are self-avoiding only over finite time intervals. This provides a conceptual simplification of the method for the selfavoiding walk, and is also significant for further application of lace expansion methods to branched polymers, for which it is less clear how to implement a finite memory [19].

The self-avoiding walk is defined and reviewed in the next section. Then our results are stated in Sect. 1.2. The organization of this paper and its relation to Part II is described in Sect. 1.3, and an overview of the proof of the main results is given in Sect. 1.4.

The results of this paper and Part II were announced in [18].

1.1. The Self-Avoiding Walk. An $n$-step self-avoiding walk $\omega$ on the $d$-dimensional hypercubic lattice $\mathbf{Z}^{d}$ is an ordered set $\omega=(\omega(0), \omega(1), \ldots, \omega(n))$ in $\mathbf{Z}^{d}$, with each $\omega(i) \in \mathbf{Z}^{d},|\omega(i)-\omega(i+1)|=1$, and $\omega(i) \neq \omega(j)$ for $i \neq j$. The notation $|\cdot|$ denotes Euclidean distance. Unless otherwise indicated, we take $\omega(0)=0$. We denote by $c_{n}$ the number of $n$-step self-avoiding walks, and for $x \in \mathbf{Z}^{d}$ we denote by $c_{n}(x)$ the number of $n$-step self-avoiding walks for which $\omega(n)=x$. By convention, $c_{0}=1$ and $c_{0}(x)=\delta_{x, 0}$. When $x$ is a nearest neighbour of the origin $c_{n}(x)$ counts the number of self-avoiding polygons. The mean square displacement $\left\langle|\omega(n)|^{2}\right\rangle_{n}$ is by definition the average value of the square of the distance from the origin after $n$ steps, i.e.,

$$
\left\langle|\omega(n)|^{2}\right\rangle_{n}=\frac{1}{c_{n}} \sum_{\omega:|\omega|=n}|\omega(n)|^{2},
$$

where the sum is over all $n$-step self-avoiding walks.

The conjectured asymptotic behaviour of these quantities as $n \rightarrow \infty$ is

$$
\begin{gathered}
c_{n} \sim A \mu^{n} n^{\gamma-1}, \\
\left\langle|\omega(n)|^{2}\right\rangle_{n} \sim D n^{2 v},
\end{gathered}
$$

and

$$
c_{n}(x) \sim B \mu^{n} n^{\alpha_{\text {sing }}-2},
$$

where in (1.4) $n$ and $\|x\|_{1}$ have the same parity. Any reasonable interpretation of the symbol $\sim$ may be taken at this point; we will be more precise about this notation in the next section. The constants $A, D, B$ are dimension dependent, and $\mu$ is a dimension dependent constant known as the connective constant. In all dimensions it is believed that the critical exponents satisfy the hyperscaling relation $\alpha_{\text {sing }}-2=-d v$. The critical exponent $\gamma$ is believed to take the values $43 / 32$ for $d=2,1.162 \ldots$ for $d=3$, and 1 for $d \geqq 4$, with a logarithmic correction when $d=4$. The conjectured values for $v$ are $3 / 4$ for $d=2,0.59 \ldots$ for $d=3$, and $1 / 2$ for $d \geqq 4$, again with logarithmic corrections in four dimensions. These conjectures are based on nonrigorous renormalization group arguments [30,31] and numerical work (see e.g. $[12,28]$ and references therein).

It is known that $\mu \equiv \lim _{n \rightarrow \infty} c_{n}^{1 / n}$ exists and that $c_{n} \geqq \mu^{n}[13]$. The best general upper bounds on $c_{n}$ are of the form $c_{n} \leqq \mu^{n} \exp \left[O\left(n^{2 /(d+2)} \log n\right)\right]$, with the $\log n$ not 
present for $d=2[14,22]$. There is no general proof that $v \geqq 1 / 2$ or that $v \leqq 1-\varepsilon$ for some $\varepsilon>0$. In high dimensions, it has been proved that for $d \geqq d_{0}$, for some undetermined dimension $d_{0}$, that (1.2) holds with $\gamma=1$ [34] and (1.3) holds with $v=1 / 2$ [32]. Progress is being made in the rigorous study of the weakly selfavoiding walk in four dimensions, using renormalization group methods [7, 3].

We introduce the generating functions for $c_{n}$, the susceptibility:

$$
\chi(z)=\sum_{n=0}^{\infty} c_{n} z^{n}
$$

and for $c_{n}(x)$, the two-point function:

$$
G_{z}(x)=\sum_{n=0}^{\infty} c_{n}(x) z^{n}
$$

Both of these power series have radius of convergence equal to the critical point $z_{c}=\mu^{-1}$. The correlation length of order two is defined by

$$
\xi_{2}(z)=\left[\frac{\sum_{x}|x|^{2} G_{z}(x)}{\sum_{x} G_{z}(x)}\right]^{1 / 2} .
$$

For $z \in\left(0, z_{c}\right)$, it is known that the two-point function decays exponentially as $|x| \rightarrow \infty$, and that the correlation length

$$
\xi(z)=\left(-\lim _{n \rightarrow \infty} n^{-1} \log G_{z}((n, 0, \ldots, 0))\right)^{-1}
$$

exists and diverges to infinity as $z \nearrow z_{c}$ [9]. It is widely believed that $\xi(z)$ and $\xi_{2}(z)$ are asymptotic to multiples of $\left(z_{c}-z\right)^{-v}$ as $z \nearrow z_{c}$, with the same critical exponent $v$ as that governing the mean square displacement in (1.3). It is also believed that at the critical point the two-point function decays via a power law:

$$
G_{z_{c}}(x) \sim \frac{\text { const. }}{|x|^{d-2+\eta}}
$$

with $\eta$ satisfying Fisher's scaling law $\gamma=(2-\eta) v$. Inserting $\gamma=1, v=1 / 2$ gives $\eta=0$ for $d \geqq 5$. In terms of the Fourier transform

$$
\hat{G}_{z}(k)=\sum_{x \in \mathbf{Z}^{d}} G_{z}(x) e^{i k \cdot x}, \quad k \in[-\pi, \pi]^{d},
$$

(1.9) leads one to expect

$$
\hat{G}_{z_{c}}(k) \sim \text { const. } \frac{1}{k^{2-\eta}} \text { as } k \rightarrow 0 .
$$

The simple random walk analogue of $\hat{G}_{z}(k)$ is $[1-2 d z \hat{D}(k)]^{-1}$, where $\hat{D}(k)=d^{-1} \sum_{j=1}^{d} \cos k_{j}$. At the simple random walk critical point $z=(2 d)^{-1}$, this is asymptotic to $2 d k^{-2}$ as $k \rightarrow 0$.

1.2. Main Results. Our results are stated in the following theorems.

Theorem 1.1. For $d \geqq 5$ there are positive constants $A, D$ such that the following hold. 
(a) $c_{n}=A \mu^{n}\left[1+O\left(n^{-\varepsilon}\right)\right]$ as $n \rightarrow \infty$, for any $\varepsilon<1 / 2$.

(b) $\left\langle|\omega(n)|^{2}\right\rangle_{n}=\operatorname{Dn}\left[1+O\left(n^{-\varepsilon}\right)\right]$ as $n \rightarrow \infty$, for any $\varepsilon<1 / 4$.

For $d=5, A \in[1,1.493]$ and $D \in[1.098,1.803]$.

A corollary of (a) is that $\lim _{n \rightarrow \infty} c_{n+1} / c_{n}=\mu$. This is believed to be true in all dimensions, but remains unproved for $d=2,3,4$. (Kesten has proved that $\lim _{n \rightarrow \infty} c_{n+2} / c_{n}=\mu^{2}$ in all dimensions [21].) Our method first controls the susceptibility and correlation length of order two, and then uses contour integration to prove Theorem 1.1. The results for $\chi$ and $\xi_{2}$ are stated in the next theorem. In the statement of the theorem and throughout the remainder of the paper, the notation $f(z) \sim g(z)$ means $\lim _{z \lambda_{c}} f(z) / g(z)=1$ (and similarly $a_{n} \sim b_{n}$ means $\left.\lim _{n \rightarrow \infty} a_{n} / b_{n}=1\right)$.

Theorem 1.2. For $d \geqq 5$,

$$
\chi(z) \sim \frac{A z_{c}}{z_{c}-z}
$$

and

$$
\xi_{2}(z) \sim\left(\frac{D z_{c}}{z_{c}-z}\right)^{1 / 2}
$$

as $z \nearrow z_{c}$, with the same constants $A, D$ as in Theorem 1.1 .

We have not succeeded in proving a bound of the form $c_{n}(x) \leqq B \mu^{n} n^{-d / 2}$ for $d=5$, although such a bound can be proven for a spread-out self-avoiding walk for $d>4$ [27]. Here we prove a bound on the generating function for $c_{n}(x)$ which is consistent with such an upper bound on $c_{n}(x)$. (See [26] for a related result for $x$ a nearest neighbour of the origin, for general $d$.)

Theorem 1.3. For $d \geqq 5$, $\sup _{x} \sum_{n=0}^{\infty} n^{a} c_{n}(x) \mu^{-n}<\infty$, for any $a<(d-2) / 2$.

For the correlation length we have the following result. The proof is based on the method employed in [15] to study the analogous problem for percolation.

Theorem 1.4. For $d \geqq 5$,

$$
\xi(z) \sim \sqrt{\frac{D}{2 d}}\left(\frac{z_{c}}{z_{c}-z}\right)^{1 / 2} \text { as } z \nearrow z_{c} .
$$

By Theorems 1.1, 1.2 and 1.4, the length scales defined by the mean square displacement, the correlation length of order two, and the correlation length are as expected all governed by the same critical exponent $v=1 / 2$. Moreover, if we set $D=1$ and $z_{c}=(2 d)^{-1}$ in the asymptotic forms of these three quantities, then we exactly recover the asymptotic forms of their simple random walk analogues.

Our results for the critical two-point function are stronger in $k$-space than in $x$-space, and are summarized in the following theorem. The upper bound on $G_{z_{c}}(x)$ in the theorem, for $p<(d-2) / 2$, follows immediately from Theorem 1.3 and the fact that $|x|^{p} c_{n}(x) \leqq n^{p} c_{n}(x)$. The $k$-space result provides a strong infrared bound.

Theorem 1.5. Let $d \geqq 5$. For any $p$ satisfying $p<(d-2) / 2$ or $p \leqq 2$ there is a constant $C(p)$ such that for all $x, G_{z_{c}}(x) \leqq C(p)|x|^{-p}$. The Fourier transform satisfies $\hat{G}_{z_{c}}(k)=$ const. $\left[k^{2}+O\left(k^{2+\varepsilon}\right)\right]^{-1}$ as $k \rightarrow 0$, for any $\varepsilon<1 / 2$. 
To discuss the scaling limit we first introduce some notation. Let $C_{d}[0,1]$ denote the continuous $\mathbf{R}^{d}$-valued functions on $[0,1]$. Given an $n$-step self-avoiding walk $\omega$, we define $X_{n} \in C_{d}[0,1]$ by taking $X_{n}(t)$ to be the linear interpolation of the points $n^{-1 / 2} \omega([n t])$, where $[n t]$ denotes the integer part of $n t$. We denote by $d W$ the Wiener measure on $C_{d}[0,1]$, unconventionally normalized so that $\int e^{i k \cdot B_{t}} d W=\exp \left[-D k^{2} t / 2 d\right]$, where $D$ is the diffusion constant of Theorem 1.1. Expectation with respect to the uniform measure on the $n$-step self-avoiding walks is denoted by $\langle\cdot\rangle_{n}$. The following theorem extends the results of $[33,34]$ from $d \geqq d_{0}$ to $d \geqq 5$.

Theorem 1.6. For $d \geqq 5$, the self-avoiding walk converges in distribution to Brownian motion. In other words for any bounded continuous function $f$ on $C_{d}[0,1]$,

$$
\lim _{n \rightarrow \infty}\left\langle f\left(X_{n}\right)\right\rangle_{n}=\int f d W .
$$

In [24], the lace expansion was used to construct the infinite self-avoiding walk for $d \geqq d_{0}$. This construction can be extended using the methods of this paper to $d \geqq 5$. See Sect. 5 for the definition of the infinite self-avoiding walk. We have no reason to doubt that the infinite self-avoiding walk exists in all dimensions, but a new idea will be needed to prove its existence for $d=2,3,4$.

Theorem 1.7. The infinite self-avoiding walk exists for $d \geqq 5$.

All of the above results rely on the lace expansion. In the course of the proof of Theorem 1.2, good bounds on the value of $\mu$ were required. An elementary method of obtaining a lower bound on $\mu$ was developed, which does not use the lace expansion, and which is valid above two dimensions. The resulting bound in three dimensions slightly improves the previous best rigorous lower bound 4.352 of [11]. The bounds for $d=3,4,5$ are as given in the following theorem. The proof of this theorem is independent of the rest of our methods, and can be found in Appendix A of Part II.

Theorem 1.8. Let $\mu(d)$ denote the connective constant for $\mathbf{Z}^{d}$. Then $\mu(3) \geqq 4.43733$, $\mu(4) \geqq 6.71800$, and $\mu(5) \geqq 8.82128$.

1.3. Organization. The proof of the results stated in Sect. 1.2 is long and divides naturally into two parts. This paper treats one part of the proof, while the second is treated in Part II. Part II contains all aspects of the proof which involve numerical computations. In this section we give a brief guide to the organization of this paper and its relation to Part II. Equation, section and theorem numbers from Part II are indicated with the prefix II.

Section 1.4 gives an overview of some of the main ideas involved in the proofs of the theorems stated in Sect. 1.2.

Sections 2.1 and 2.2 give a general description of the lace expansion and a review of the method of [8] for obtaining bounds on the expansion. However for $d=5$ much more detailed methods are required for proving convergence of the expansion. These methods are presented in Part II, where the proof of convergence of the lace expansion is given. One ingredient of the convergence proof is the bound on the connective constant of Theorem 1.8. The proof of Theorem 1.8 is given in Appendix A of Part II. 
In Sect. 2.3 we summarize the results of Part II which are needed to prove Theorems 1.1-1.7. Theorem 1.2 follows immediately from the results summarized in Sect. 2.3; its proof is given in Sect. 3.1.

In addition to the estimates from Part II summarized in Sect. 2.3, the proofs of Theorems 1.1 and 1.3-1.7 rely on the fact that certain norms of "fractional derivatives" of the propagator $G_{z}(x)$ are finite at the critical point $z=z_{c}$. A calculus of fractional derivatives is briefly developed in Sect. 3.2, and then applied to the propagator in Sect. 3.3, where Theorem 1.3 is proved. Then contour integration is used to prove Theorem 1.1 in Sect. 3.4. However the proof of the numerical bounds on $A$ and $D$ for $d=5$ relies on numerical estimates and is deferred to the end of Sect. II.1.3. Section 3.5 contains the proof of Theorem 1.4.

The infrared bound is intimately connected with the convergence of the expansion, and in Corollary II.1.2 upper and lower bounds are obtained on $\hat{G}_{z_{c}}(k)$. These bounds are then improved to give the bound on $\hat{G}_{z_{c}}(k)$ of Theorem 1.5 in Sect. 3.6. The $x$-space bound $G_{z_{c}}(x) \leqq$ const. $|x|^{-2}$ of Theorem 1.5 is proved in Theorem II.1.1.

In Sect. 4 the proof of Theorem 1.6 is given. Finally in Sect. 5 we prove Theorem 1.7.

1.4. Overview of the Method. In this section we give a brief general overview of the methods used to prove the results for $c_{n}$ and $\chi(z)$ stated in Theorems 1.1 and 1.2. The estimates and methodology developed in proving these results illustrate many features of the proofs of the other theorems.

The basic starting point is the lace expansion, which provides a formula for the quantity $\hat{\Pi}_{z}(k)$ defined implicitly by the equation

$$
\hat{G}_{z}(k)=\frac{1}{1-2 d z \hat{D}(k)-\hat{\Pi}_{z}(k)} .
$$

The lace expansion can be derived either by an expansion and resummation procedure similar to that used to derive cluster expansions in statistical mechanics and quantum field theory $[8,6]$, or by repeated application of the inclusionexclusion relation [35]. The resulting formula for $\hat{\Pi}_{z}(k)$ can be bounded above, using the repulsive nature of the self-avoidance interaction, by a sum of Feynman diagrams in which the lines in the diagram represent the propagator (two-point function) (1.12). It is then possible to estimate these diagrams to obtain an upper bound on $\hat{\Pi}_{z}(k)$ in terms of the two-point function itself. In particular, a bound on the zeroth, first and second derivatives of $\hat{\Pi}_{z}(k)$ with respect to $k_{\mu}$ can be obtained of the form

$\left|\partial_{\mu}^{u} \hat{\Pi}_{z}(k)\right| \leqq\left\|x_{\mu}^{u} G_{|z|}(x)\right\|_{\infty}\left[\delta_{0, u} 2 d|z|+\sum_{N=2}^{\infty} \frac{N}{2} B(|z|)^{N / 2}[1+B(|z|)]^{(N-2) / 2}\right]$

$(u=0,1,2)$, where $B(z)$ is the "bubble diagram"

$$
B(z)=\sum_{x \neq 0} G_{z}(x)^{2}
$$

and the norm in (1.13) is the supremum norm. Bounds such as (1.13) can be somewhat costly in numerical terms, and considerably more elaborate estimates are used in Part II. 
Convergence of the right side of (1.13) requires that $B(|z|)[1+B(|z|)]<1$. For simple random walk at its critical point $z=(2 d)^{-1}$, the bubble diagram is infinite for $d \leqq 4$ and diverges as $d \rightarrow 4^{+}$. The same can be expected for the self-avoiding walk. This restricts the method to $d>4$, or more accurately to $d$ larger than four plus some positive amount. In this sense our method is unnatural. A more natural method would have as its driving force the fact that $B\left(z_{c}\right)$ is finite rather than small, but unfortunately such a method has not materialized. For $d=5$ we expect $B\left(z_{c}\right)$ to be somewhat less than the critical simple random walk bubble diagram value of 0.5979 . Since $(0.5979)(1.5979)=0.9554<1$, the required inequality is satisfied with this value, but barely.

The estimate (1.13) provides a bound on $\partial_{\mu}^{u} \hat{\Pi}_{z}(k)$ in terms of the norms $B(z)^{1 / 2}$ and $\left\|x_{\mu}^{u} G_{z}(x)\right\|_{\infty}$. But (1.12) provides a means of calculating these norms in terms of $\partial_{\mu}^{u} \hat{\Pi}_{z}(k)$ itself. It was shown in [32] that in high dimensions self-consistency requires a finite uniform bound on these two norms, for $|z| \leqq z_{c}$. This gave the $x$-space bound asserted in Theorem 1.5 (with $p \leqq 2$ ) for high dimensions, and in the course of establishing these bounds an infrared bound was also obtained. In Part II this self-consistency argument is improved to $d \geqq 5$. Dealing with actual $d=5$ values, rather than simply taking the inverse dimension as small as required, complicates the proof considerably. Computer calculations played an important experimental role in developing the proof, and in performing some of the calculations in the final version. Rigorous error bounds were obtained for the computer calculations.

Similar arguments lead to a proof that $\left\|\partial_{z} G_{|z|}(x)\right\|_{\infty}$ is finite, and a finite bound on $\left|\partial_{z} \hat{\Pi}_{z}(k)\right|$ in terms of this norm, for $|z| \leqq z_{c}$. Higher order $z$-derivatives of $\hat{\Pi}_{z}(k)$ would involve $l^{\infty}$ norms of higher order derivatives of $G_{z}(x)$.

A basic philosophy underlying the analysis is that for $d$ greater than four $\hat{G}_{z_{c}}(k)$ behaves essentially like the critical simple random walk two-point function $\hat{C}(k) \equiv[1-\hat{D}(k)]^{-1}$. According to this philosophy, $\partial_{z}^{2} \hat{\Pi}_{z_{c}}(k)$ should be infinite for $d \leqq 6$ (so in particular for $d=5$ ) since for simple random walk the norm $\left\|\partial_{z}^{2} C(x)\right\|_{\infty}$ behaves like $\sum_{n} n^{2} n^{-d / 2}$. However for $d>4$ it should be possible to take fractionally more than one derivative, since $\sum_{n} n^{1+\varepsilon} n^{-d / 2}<\infty$ for $\varepsilon<(d-4) / 2$. In Sect. 3.2 a method of calculating such "fractional derivatives" is developed, and it is then applied in Sect. 3.3 to prove that $\left|\delta_{z}^{\varepsilon} \partial_{z} \hat{\Pi}_{z_{c}}(k)\right|<\infty\left(\delta_{z}^{\varepsilon}\right.$ is defined in Sect. 3.2).

It follows from the fact that the bubble diagram is finite at the critical point that $\chi(z)$ is bounded above and below by positive multiples of $\left(z_{c}-z\right)^{-1}$ as $z \nearrow z_{c}$ [5]. To obtain the stronger asymptotic behaviour claimed in Theorem 1.2, we first note that because $\hat{G}_{z_{c}}(0)^{-1}=1-2 d z_{c}-\hat{\Pi}_{z_{c}}(0)=0$,

$$
\chi(z)=\hat{G}_{z}(0)=\frac{1}{\hat{G}_{z}(0)^{-1}-\hat{G}_{z_{c}}(0)^{-1}}=\frac{1}{2 d\left(z_{c}-z\right)+\hat{\Pi}_{z_{c}}(0)-\hat{\Pi}_{z}(0)} .
$$

The desired asymptotic behaviour

$$
\chi(z) \sim\left[2 d+\partial_{z} \hat{\Pi}_{z_{c}}(0)\right]^{-1}\left(z_{c}-z\right)^{-1}
$$

then follows from a positive lower bound on $2 d+\partial_{z} \hat{\Pi}_{z_{c}}(0)$, which is itself established in the self-consistency argument referred to above.

To prove Theorem 1.1(a), we now want to obtain the large- $n$ asymptotics of $c_{n}$ from the asymptotic behaviour (1.16) of the generating function at its closest 
singularity to the origin. Generally additional information beyond (1.16) is required for such a task, and any additional sufficient condition usually is called a Tauberian condition. To see what sort of error term is involved, we add and subtract the right side of (1.16) to (1.15), and perform a contour integral to obtain $c_{n}$. The result can be written as

$$
c_{n}=\left[2 d+\partial_{z} \hat{\Pi}_{z_{c}}(0)\right]^{-1} z_{c}^{-n-1}\left[1+\frac{1}{2 \pi i} \oint \frac{h(z)}{\left(z_{c}-z\right)} \chi(z)\left(\frac{z_{c}}{z}\right)^{n+1} d z\right],
$$

where

$$
h(z)=\partial_{z} \hat{\Pi}_{z_{c}}(0)\left(z_{c}-z\right)-\hat{\Pi}_{z_{c}}(0)+\hat{\Pi}_{z}(0)
$$

and the contour is a small circle centred at the origin. We now proceed to analyze the error term, i.e., the contour integral on the right side of (1.17).

If the contour is deformed to the circle of convergence $|z|=z_{c}$, the factor $\left(z_{c} / z\right)^{n+1}$ becomes $e^{-i(n+1) \theta}$. If the rest of the integrand were integrable on the circle $|z|=z_{c}$, then by the Riemann-Lebesgue lemma the error term would go to zero as $n \rightarrow \infty$. As discussed above, it can be shown that $(1+\varepsilon) z$-derivatives of $\hat{\Pi}_{z}(k)$ can be taken. A lemma in the spirit of Taylor's theorem then can be used to show that $h(z)$ vanishes at least as fast as $\left|z_{c}-z\right|^{1+\varepsilon}$ at $z_{c}$, which is enough to give integrability. We also prove a lemma which gives control of the error in this type of Riemann-Lebesgue argument.

\section{The Lace Expansion}

Sections 2.1 and 2.2 provide a brief overview of the Brydges-Spencer lace expansion. Readers already familiar with the lace expansion may wish to skip directly to Sect. 2.3. A detailed account of the lace expansion is given in [27].

2.1. General Description. We first need to introduce some notation and terminology. Given an $n$-step simple random walk $\omega=(\omega(0), \omega(1), \ldots, \omega(n))$ and two nonnegative integers $s$ and $t$, we define

$$
\mathscr{U}_{s t}(\omega)=\left\{\begin{aligned}
-1 & \text { if } \omega(s)=\omega(t) \\
0 & \text { if } \omega(s) \neq \omega(t)
\end{aligned}\right.
$$

The self-avoiding walk two-point function can then be written

$$
G_{z}(x)=\sum_{\omega: 0 \rightarrow x} z^{|\omega|} \prod_{0 \leqq s<t \leqq|\omega|}\left(1+\mathscr{U}_{s t}(\omega)\right),
$$

where the sum over $\omega$ is the sum over all simple random walks ending at $x$.

The Fourier transform of (2.2) is given by

$$
\hat{G}_{z}(k)=\sum_{\omega} z^{|\omega|} e^{i k \cdot \omega(|\omega|)} \prod_{0 \leqq s<t \leqq|\omega|}\left(1+\mathscr{U}_{s t}(\omega)\right) .
$$

We denote the radius of convergence of the right side by $z_{c}(k)$. Since $\hat{G}_{z}(0)=\chi(z)$, $z_{c}(0)=z_{c}$. For any $k$,

$$
z_{c}(k) \geqq z_{c} .
$$


To obtain a formula for the inverse of the propagator $\hat{G}_{z}(k)$, we first introduce some terminology.

Definition 2.1. Given an interval $I=[a, b]$ of positive integers, we refer to a pair $s t$ of elements of $I$, with $s<t$, as an edge. The length of an edge $s t$ is $t-s$. A set of edges is called a graph. A graph $\Gamma$ is said to be connected if both $a$ and $b$ are endpoints of edges in $\Gamma$, and if in addition for any $c \in(a, b)$ there are $s, t \in[a, b]$ such that $s<c<t$ and $s t \in \Gamma$. The set of all graphs on $[a, b]$ is denoted $\mathscr{B}[a, b]$, and the subset consisting of all connected graphs is denoted $\mathscr{G}[a, b]$. A lace is a minimally connected graph, i.e., a connected graph for which the removal of any edge would result in a disconnected graph. The set of laces on $[a, b]$ is denoted by $\mathscr{L}[a, b]$, and the set of laces on $[a, b]$ which consist of exactly $N$ edges is denoted $\mathscr{L}_{N}[a, b]$.

The following prescription associates to each connected graph $\Gamma$ a unique lace $\mathscr{L}_{\Gamma}$. The lace $\mathscr{L}_{\Gamma}$ consists of edges $s_{1} t_{1}, s_{2} t_{2}, \ldots$ where

$$
\begin{aligned}
s_{1} & =a, \quad t_{1}=\max \{t: a t \in \Gamma\}, \\
t_{i+1} & =\max \left\{t: s t \in \Gamma, s<t_{i}\right\}, \\
s_{i} & =\min \left\{s: s t_{i} \in \Gamma\right\} .
\end{aligned}
$$

Given a lace $L$, the set of all edges $s t \notin L$ such that $\mathscr{L}_{L \cup\{s t\}}=L$ is denoted $\mathscr{C}(L)$. Edges in $\mathscr{C}(L)$ are said to be compatible with $L$.

For $a<b$ we define

$$
K[a, b]=\prod_{s t \in \mathscr{B}[a, b]}\left(1+\mathscr{U}_{s t}\right) .
$$

We set $K[a, a]=1$. For $a<b$ we also define

$$
J_{N}[a, b]=\sum_{L \in \mathscr{L}_{N}[a, b]} \prod_{s t \in L} \mathscr{U}_{s t} \prod_{s^{\prime} t^{\prime} \in \mathscr{C}(L)}\left(1+\mathscr{U}_{s^{\prime} t^{\prime}}\right)
$$

and

$$
J[a, b]=\sum_{N=1}^{\infty} J_{N}[a, b] .
$$

The sum in (2.7) is a finite sum, since the sum in (2.6) is empty for $N>b-a$. We set $J[a, a]=1$. By definition, $J[a, a+1]=0$, since the only lace on $[a, a+1]$ consists of the single edge $\{a, a+1\}$ and $\mathscr{U}_{a, a+1}=0$ (because a walk cannot be at the same place at consecutive times).

Now we define

$$
\Pi_{z}^{(N)}(x)=(-1)^{N} \sum_{\substack{\omega: 0 \rightarrow x \\|\omega| \geqq 2}} z^{|\omega|} J_{N}[0,|\omega|]
$$

and

$$
\Pi_{z}(x)=\sum_{N=1}^{\infty}(-1)^{N} \Pi_{z}^{(N)}(x)=\sum_{\substack{\omega: 0 \rightarrow x \\|\omega| \geqq 2}} z^{|\omega|} J[0,|\omega|],
$$

for any $z$ for which the right sides converge. By definition, $\Pi_{z}^{(N)}(x) \geqq 0$ for all nonnegative $z$. Theorem 2.6 below asserts that for $d \geqq 5$,

$$
\sum_{\omega:|\omega| \geqq 2} z_{c}^{|\omega|}|J[0,|\omega|]|<\infty \text {. }
$$


Equation (2.10) guarantees the existence of the Fourier transform of $\Pi_{z}(x)$, for $|z| \leqq z_{c}$. Recall that $\hat{D}(k)=d^{-1} \sum_{\mu=1}^{d} \cos k_{\mu}$.

Theorem 2.2. (Brydges and Spencer [8]). For any value of $z$ for which $\sum_{|\omega| \geqq 2} z^{|\omega|} J[0,|\omega|]$ and $\sum_{|\omega| \geqq 0} z^{|\omega|} K[0,|\omega|]$ converge absolutely,

$$
G_{z}(x)=\delta_{0, x}+z \sum_{y:|y|=1} G_{z}(x-y)+\sum_{v \in \mathbf{Z}^{a}} \Pi_{z}(v) G_{z}(x-v)
$$

and

$$
\hat{G}_{z}(k)=\frac{1}{1-2 d z \hat{D}(k)-\hat{\Pi}_{z}(k)} .
$$

Theorem 2.2 gives an expression for the inverse of the propagator $\hat{G}_{z}(k)$. By definition,

$$
\hat{\Pi}_{z}(k)=\sum_{N=1}^{\infty} \sum_{\omega:|\omega| \geqq 2} e^{i k \cdot \omega(|\omega|)} z^{|\omega|} J_{N}[0,|\omega|] .
$$

There is a useful diagrammatic interpretation for the terms in the above sum over $N$, arising from the fact that $J_{N}[0,|\omega|] \neq 0$ only for walks $\omega$ with a specific topology. This has been described in detail in [8], so we state this interpretation without detailed explanations here. The factor $\prod_{s t \in L} \mathscr{U}_{s t}$ in (2.6) is nonzero only if the walk intersects itself at times $s$ and $t$, for each $s t \in L$, while the factor $\prod_{s t \in \mathscr{G}(L)}\left(1+\mathscr{U}_{s t}\right)$ rules out many (but in general not all) other self-intersections. The walk decomposes in a natural way into $2 N-1$ strictly self-avoiding subwalks, corresponding to the time intervals intervening between the intersections required for $\prod_{s t \in L} \mathscr{U}_{s t} \neq 0$. We represent the $N^{\text {th }}$ term: $(-1)^{N} \hat{\Pi}_{z}^{(N)}(k)$ of (2.13) by an $N$-loop diagram, as follows:

$$
\hat{\Pi}_{z}(k)=-0+\emptyset-\mathbb{D}+\mathbb{D}-\cdots
$$

The subwalks which are not slashed in (2.14) must consist of at least one step, whereas the slashed subwalks can be of zero or more steps. The subwalks forming each loop of a diagram are mutually avoiding. Further mutual avoidance is also present.

2.2. Bounds on the Lace Expansion. To bound a term on the right side of (2.14), we first take absolute values inside all sums. We then overcount by neglecting all mutual avoidance between distinct subwalks. To give an explicit example, we first define

$$
G_{z}^{(1)}(x)=\sum_{n=1}^{\infty} c_{n}(x) z^{n}=G_{z}(x)-\delta_{0, x} .
$$

Then the three loop diagram is bounded above in absolute value by

$$
\sum_{x, y} G_{|z|}^{(1)}(y)^{2} G_{|z|}(x) G_{|z|}^{(1)}(x-y)^{2} .
$$

Brydges and Spencer [8] proved the following lemma, which can be used to bound diagrams such as those occurring in (2.14), or their derivatives with respect to $z$ and $k$. Although this lemma will be adequate for some of our needs, we will require considerably more detailed estimates on some diagrams, particularly those with few loops. These detailed estimates will appear in Part II. 
Lemma 2.3. Evaluate the $N$-loop diagram contributing to the right side of (2.14) by associating to any one vertex the origin in $\mathbf{Z}^{d}$, associating to each additional vertex an index in $\mathbf{Z}^{d}$, and associating to the $i^{\text {th }}$ subwalk an even function $f_{i}$ evaluated at the difference of the indices corresponding to the endpoints of the subwalk, and then summing the product of the $f_{i}$ 's over all indices. Then for any $j \in\{1, \ldots, 2 N-1\}$ the diagram is bounded above by

$$
\left\|f_{j}\right\|_{\infty} \prod_{i \neq j}\left\|f_{i}\right\|_{2},
$$

where $\|\cdot\|_{p}$ denotes the $l^{p}$ norm for functions on $\mathbf{Z}^{d}$.

This lemma can be used directly to estimate expressions such as (2.16). It can also be used to estimate various modifications of the diagrams in (2.14), and this is described in detail in [8]. Here we try to give an idea of how the process works. Given a nonnegative integer $u$, if we take the $u^{\text {th }} k_{\mu}$-derivative of a diagram, we can bound the result by introducing a factor $\left|x_{\mu}\right|^{u}$. Similarly a $z$-derivative introduces a factor of $|\omega|$. More generally we consider the case in which a factor $|\omega|^{v}$ is present, where $v$ is a positive real number. Denoting the subwalks in the $N$-loop diagram by $\omega_{i}$, we can write $|\omega|=\sum_{i=1}^{2 N-1}\left|\omega_{i}\right|$, and thus by an appropriate inequality (e.g. $|\omega|^{v} \leqq(2 N-1)^{v-1} \sum_{i=1}^{2 N-1}\left|\omega_{i}\right|^{v}$ for $\left.v \geqq 1\right)$ we obtain a sum of diagrams in which one subwalk carries a factor $\left|\omega_{i}\right|^{v}$. Similarly, if $\left|x_{\mu}\right|^{u}$ is present, we write $x_{\mu}=\sum_{i} y_{\mu}^{(i)}$ as the sum of displacements along a number of subwalks, and with an appropriate inequality we can arrange that in a particular term only one subwalk carries a factor $\left|y_{\mu}^{(i)}\right|^{u}$. Moreover if both $|\omega|^{v}$ and $\left|y_{\mu}^{(i)}\right|^{u}$ are present, it can be arranged that different subwalks carry the factors $\left|y_{\mu}^{(j)}\right|^{u}$ and $\left|\omega_{i}\right|^{v}$. The number of terms obtained will be polynomial in the number of loops. All other subwalks will be unchanged. Reasoning in this way, the following lemma can be proved. See for example $[8,27]$; more detailed estimates are obtained in Part II.

Lemma 2.4. Suppose that in each diagram contributing to (2.14) the factor $|x|^{u}|\omega|^{v}$ is introduced. The result is bounded absolutely by a finite constant if

$$
\left\||x|^{u} G_{|z|}(x)\right\|_{r},\left\|\sum_{n=1}^{\infty} n^{v} c_{n}(x)|z|^{n}\right\|_{s}<\infty
$$

with $(r, s)=(2, \infty)$ or $(\infty, 2)$, and if in addition

$$
\left\|G_{|z|}(x)\right\|_{2}^{2}\left\|G_{|z|}^{(1)}(x)\right\|_{2}^{2}<1 .
$$

If these inequalities are satisfied, then the sum of modified diagrams in (2.14) is proportional to each of the norms in (2.17).

2.3. Bounds Required from Part II. In this section we collect some results from Part II which are needed in the remainder of Part I. See Theorem II.1.1 for the proofs of these results.

Theorem 2.5. Let $d \geqq 5$. There are constants $C_{1}, C_{2}$ such that for any $z$ with $|z| \leqq z_{c}$,

and

$$
\left\||x|^{2} G_{z}(x)\right\|_{\infty} \leqq C_{1}
$$

$$
\left\|G_{z}^{(1)}(x)\right\|_{2}^{2} \leqq C_{2},
$$

with $C_{2}\left(1+C_{2}\right)<1$, i.e., (2.18) is satisfied. 
Theorem 2.6. Let $d \geqq 5$ and $u \in\{0,1,2\}$. The quantities $\left|\partial_{z} \hat{\Pi}_{z}(k)\right|$ and $\left|\partial_{\mu}^{u} \hat{\Pi}_{z}(k)\right|$ are finite and bounded uniformly in $k \in[-\pi, \pi]^{d}$ and $|z| \leqq z_{c}$. In fact the series representations of these quantities are bounded absolutely (absolute values inside sums over $x, N)$ and uniformly.

It follows from Theorem 2.6 and the dominated convergence theorem that $\partial_{z} \hat{\Pi}_{z}(k)$ and $\partial_{\mu}^{u} \hat{\Pi}_{z}(k)(u=0,1,2)$ are continuous on the closed disk $|z| \leqq z_{c}$. In particular, since $\chi(z)=\left[1-2 d z-\hat{\Pi}_{z}(0)\right]^{-1}$ diverges at $z_{c}$ we have $1-2 d z_{c}$ $-\hat{\Pi}_{z_{c}}(0)=0$.

Theorem 2.7. Let $d \geqq 5$. For $z=z_{c} e^{i \theta}$ with $\theta \neq 0$, the zero-momentum inverse propagator $1-2 d z-\hat{\Pi}_{z}(0)$ is nonzero.

Theorem 2.8. Let $d \geqq 5$. There are positive constants $C_{3}, C_{4}, \varepsilon_{1}$ such that for any $p \in\left[z_{c}-\varepsilon_{1}, z_{c}\right]$

$$
2 d+\partial_{z} \hat{\Pi}_{p}(0) \geqq C_{3}>0
$$

and

$$
2 d p-\nabla_{k}^{2} \hat{\Pi}_{p}(0)=2 d p+\sum_{x}|x|^{2} \Pi_{p}(x) \geqq C_{4}>0
$$

Also, there is a positive constant $C_{5}$ such that for any $p \in\left[0, z_{c}\right]$,

$$
\hat{\Pi}_{p}(0)-\hat{\Pi}_{p}(k) \geqq-C_{5}[1-\hat{D}(k)],
$$

with $2 d z_{c}-C_{5}>0$. In particular, $\hat{F}_{z_{c}}(k) \geqq 0$, where $\hat{F}_{z}(k)=1 / \hat{G}_{z}(k)$.

\section{The Critical Exponents}

This section is organized as follows. We begin in Sect. 3.1 with the proof of Theorem 1.2. In Sect. 3.2 we prove some elementary lemmas concerning fractional derivatives. In Sect. 3.3 we prove existence of fractional derivatives of $\hat{\Pi}_{z}(k)$, and also prove Theorem 1.3 [see Theorem 3.7(a)]. In Sect. 3.4 contour integration is used to prove Theorem 1.1. In Sect. 3.5 the correlation length is studied and Theorem 1.4 is proved. Finally in Sect. 3.6 the proof of Theorem 1.5 is completed.

3.1. Proof of Theorem 1.2. The bubble diagram is defined by $B(z)=\left\|G_{z}^{(1)}(x)\right\|_{2}^{2}$. It was shown in [5] that if $B\left(z_{c}\right)<\infty$, then there are constants $c_{1}$ and $c_{2}$ such that for $p<z_{c}$,

$$
c_{1}\left(z_{c}-p\right)^{-1} \leqq \chi(p) \leqq c_{2}\left(z_{c}-p\right)^{-1} .
$$

Thus by Theorem 2.5 and the results of [5], (3.1) holds.

To obtain the stronger asymptotic behaviour stated in Theorem 1.2, we observe that since $1-2 d z_{c}-\hat{\Pi}_{z_{c}}(0)=0$,

$$
\chi(z)=\left(\frac{1}{z_{c}-z}\right)\left(2 d+\frac{\hat{\Pi}_{z_{c}}(0)-\hat{\Pi}_{z}(0)}{z_{c}-z}\right)^{-1} .
$$


It then follows from Theorem 2.8 that $\chi(z)$ is asymptotic to $\left[2 d+\partial_{z} \hat{\Pi}_{z_{c}}(0)\right]^{-1}\left(z_{c}-z\right)^{-1}$. Defining

$$
A=\frac{1}{-z_{c}\left[\partial_{z} \hat{F}_{z_{c}}(0)\right]}=\frac{1}{z_{c}\left[2 d+\partial_{z} \hat{\Pi}_{z_{c}}(0)\right]}
$$

gives the desired result.

For the correlation length of order two, we note that by symmetry and direct calculation,

$$
\xi_{2}(z)^{2}=\frac{-\nabla_{k}^{2} \hat{G}_{z}(0)}{\hat{G}_{z}(0)}=\left[2 d z-\nabla_{k}^{2} \hat{\Pi}_{z}(0)\right] \chi(z) .
$$

The desired asymptotic behaviour of $\xi_{2}(z)$ now follows from the asymptotic behaviour of $\chi(z)$ and the bound on $\nabla_{k}^{2} \hat{\Pi}_{z}(0)$ of Theorem 2.8, if we define

$$
D=A\left[2 d z_{c}-\nabla_{k}^{2} \hat{\Pi}_{z_{c}}(0)\right]=\frac{\nabla_{k}^{2} \hat{F}_{z_{c}}(0)}{z_{c}\left[-\partial_{z} \hat{F}_{z_{c}}(0)\right]} .
$$

3.2. Fractional Derivatives. In this section we describe some elementary properties of what we term fractional derivatives. This terminology is somewhat inaccurate, but is useful in a suggestive sense in the analysis of the large- $n$ asymptotics of power series coefficients. Given a power series $f(z)=\sum_{n=0}^{\infty} a_{n} z^{n}$ and $\varepsilon \geqq 0$, we define the fractional derivative

$$
\delta_{z}^{\varepsilon} f(z)=\sum_{n=0}^{\infty} n^{\varepsilon} a_{n} z^{n} .
$$

Note that for $\varepsilon$ a positive integer $\delta_{z}^{\varepsilon}$ does not give the usual derivative. We will use (3.6) with $\varepsilon \in(0,1)$. Allowing $\varepsilon$ to take on arbitrary negative values defines a relative of the antiderivative, as follows. For $\alpha>0$ we define

$$
\delta_{z}^{-\alpha} f(z)=\sum_{n=1}^{\infty} n^{-\alpha} a_{n} z^{n} .
$$

Both of the above quantities will be finite at least strictly within the circle of convergence of $f(z)$.

The following lemma provides formulas which are convenient for estimating fractional derivatives.

Lemma 3.1. Let $f(z)=\sum_{n=0}^{\infty} a_{n} z^{n}$ have radius of convergence $R$. Then for any $z$ with $|z|<R$, and for any $\alpha>0$,

$$
\delta_{z}^{-\alpha} f(z)=C_{\alpha} \int_{0}^{\infty}\left[f\left(z e^{-\lambda^{1 / \alpha}}\right)-f(0)\right] d \lambda,
$$

where $C_{\alpha}=[\alpha \Gamma(\alpha)]^{-1}$. In addition, for any $z$ with $|z|<R$ and for any $\varepsilon \in(0,1)$,

$$
\delta_{z}^{\varepsilon} f(z)=C_{1-\varepsilon} z \int_{0}^{\infty} f^{\prime}\left(z e^{-\lambda^{1 /(1-\varepsilon)}}\right) e^{-\lambda^{1 /(1-z)}} d \lambda .
$$

The identities (3.8) or (3.9) also hold for $z=R$, if $a_{n} \geqq 0$. 
Proof. Let $|z|<R$. We first note that for any $\alpha>0$,

$$
n^{-\alpha}=\frac{1}{\alpha \Gamma(\alpha)} \int_{0}^{\infty} e^{-n \lambda^{1 / \alpha}} d \lambda
$$

as can be seen by making the substitution $y=n \lambda^{1 / \alpha}$ in the integral on the right side. Therefore

$$
\sum_{n=1}^{\infty} n^{-\alpha} a_{n} z^{n}=C_{\alpha} \sum_{n=1}^{\infty} a_{n} \int_{0}^{\infty}\left(z e^{-\lambda^{1 / \alpha}}\right)^{n} d \lambda .
$$

Since the right side converges absolutely the order of integration and summation can be interchanged to yield (3.8).

For (3.9), we write $n^{\varepsilon}=n^{-(1-\varepsilon)} n$ and use (3.10) with $\alpha=1-\varepsilon$ to obtain

$$
\sum_{n=0}^{\infty} n^{\varepsilon} a_{n} z^{n}=C_{1-\varepsilon} z \sum_{n=1}^{\infty} n a_{n} \int_{0}^{\infty}\left(z e^{-\lambda^{1 /(1-\varepsilon)}}\right)^{n-1} e^{-\lambda^{1 /(1-\varepsilon)}} d \lambda .
$$

Since the right side converges absolutely we can interchange the order of summation and integration to obtain

$$
\sum_{n=0}^{\infty} n^{\varepsilon} a_{n} z^{n}=C_{1-\varepsilon} z \int_{0}^{\infty} f^{\prime}\left(z e^{-\lambda^{1 /(1-\varepsilon)}}\right) e^{-\lambda^{1 /(1-\varepsilon)}} d \lambda .
$$

Now suppose that $a_{n} \geqq 0$ and take $z=R$. Then the above interchanges of sum and integral are justified by Fubini's Theorem.

The following lemma provides an error estimate analogous to the error estimate in Taylor's theorem. In applications of the lemma $R$ will be the radius of convergence of $f$.

Lemma 3.2. Let $\varepsilon \in(0,1)$ and let $f(z)=\sum_{n=0}^{\infty} a_{n} z^{n}$. Let $R>0$ and suppose that $A_{\varepsilon} \equiv \sum_{n=0}^{\infty} n^{\varepsilon}\left|a_{n}\right| R^{n-\varepsilon}<\infty$, so in particular $f(z)$ converges for $|z| \leqq R$. Then for any $z$ with $|z| \leqq R$,

$$
|f(z)-f(R)| \leqq 2^{1-\varepsilon} A_{\varepsilon}|R-z|^{\varepsilon} .
$$

Suppose that $B_{\varepsilon} \equiv \sum_{n=1}^{\infty} n^{1+\varepsilon}\left|a_{n}\right| R^{n-1-\varepsilon}<\infty$, so in particular $f^{\prime}(z)=$ $\sum_{n=0}^{\infty} n a_{n} z^{n-1}$ converges for $|z| \leqq R$. Then for any $z$ with $|z| \leqq R$,

$$
\left|f(z)-f(R)-f^{\prime}(R)(z-R)\right| \leqq \frac{2^{1-\varepsilon}}{1+\varepsilon} B_{\varepsilon}|R-z|^{1+\varepsilon} .
$$

Proof. We just give the proof of (3.15). The proof of (3.14) is similar and simpler. By definition,

$$
f(z)-f(R)-f^{\prime}(R)(z-R)=(z-R) \sum_{n=1}^{\infty} a_{n} R^{n-1} \sum_{j=0}^{n-1}\left[\left(\frac{z}{R}\right)^{j}-1\right] .
$$

But in general

$$
\begin{aligned}
w^{j}-1 & =(w-1)^{\varepsilon}\left[\frac{w^{j}-1}{w-1}\right]^{\varepsilon}\left(w^{j}-1\right)^{1-\varepsilon} \\
& =(w-1)^{\varepsilon}\left[\sum_{m=0}^{j-1} w^{m}\right]^{\varepsilon}\left(w^{j}-1\right)^{1-\varepsilon}
\end{aligned}
$$


Taking absolute values in (3.17) and using $w=z / R$ and $|w| \leqq 1$ gives

$$
\left|\left(\frac{z}{R}\right)^{j}-1\right| \leqq|z-R|^{\varepsilon} j^{\varepsilon} 2^{1-\varepsilon} R^{-\varepsilon} .
$$

Since $\sum_{j=0}^{n-1} j^{\varepsilon} \leqq(1+\varepsilon)^{-1} n^{1+\varepsilon}$, the lemma follows from (3.16) and (3.18).

The intuition behind the following lemma is that if a power series with radius of convergence $R$ behaves like $|R-z|^{-b}$ near $z=R$, for some $b \geqq 1$, then roughly speaking it should have coefficient of $z^{n}$ not much worse than order $R^{-n} n^{b-1}$.

Lemma 3.3. Let $f(z)=\sum_{n=0}^{\infty} a_{n} z^{n}$ have radius of convergence greater than or equal to $R>0$.

(i) Suppose that for $|z|<R,|f(z)| \leqq$ const. $|R-z|^{-b}$ for some $b \geqq 1$. Then $\left|a_{n}\right|$ $\leqq O\left(R^{-n} n^{\alpha}\right)$, for any $\alpha>b-1$.

(ii) If for some $b \geqq 1$ a bound on the derivative of the form $\left|f^{\prime}(z)\right| \leqq$ const. $|R-z|^{-b}$ holds for every $|z|<R$, then $\left|a_{n}\right| \leqq O\left(R^{-n} n^{-\alpha}\right)$ for any $\alpha<2-b$.

Proof. (i) Fix $b \geqq 1$ and let $\alpha>b-1$. Since $n^{-\alpha} a_{n}$ is the coefficient of $z^{n}$ in the fractional antiderivative $\delta_{z}^{-\alpha} f(z)$,

$$
n^{-\alpha} a_{n}=\frac{1}{2 \pi i} \oint \delta_{z}^{-\alpha} f(z) \frac{d z}{z^{n+1}},
$$

where the integral is around a circle of radius $r<R$ centred at the origin. By Lemma 3.1,

$$
n^{-\alpha}\left|a_{n}\right| \leqq \text { const. } r^{-n} \int_{-\pi}^{\pi} d \theta \int_{0}^{\infty} d \lambda\left|f\left(r e^{i \theta} e^{-\lambda^{1 / \alpha}}\right)-f(0)\right|
$$

Since $f(z)-f(0)=O(|z|)$ for $z$ near zero, the contribution to the integral with respect to $\lambda$ due to $\lambda \in[1, \infty)$ is finite. Using the assumed bound on $f(z)$, we thus have

$$
n^{-\alpha}\left|a_{n}\right| \leqq \text { const. } r^{-n}\left[1+\int_{-\pi}^{\pi} d \theta \int_{0}^{1} d \lambda\left|R-r e^{i \theta} e^{-\lambda^{1 / \alpha}}\right|^{-b}\right] .
$$

Replacing the $R$ on the right side by $r$ gives an upper bound. Taking the limit $r \rightarrow R$ in the upper bound leads to

$$
n^{-\alpha}\left|a_{n}\right| \leqq \text { const. } R^{-n-b}\left[1+\int_{-\pi}^{\pi} d \theta \int_{0}^{1} d \lambda\left|1-e^{i \theta} e^{-\lambda^{1 / \alpha}}\right|^{-b}\right] .
$$

To check that the integral on the right side is finite, it suffices to show that the corresponding quantity with limits of integration $\theta= \pm 1$ is finite (or any other small finite interval containing $\theta=0$ ). Thus it suffices to verify that

$$
\int_{0}^{1} d \theta \int_{0}^{1} d \lambda\left|1-e^{i \theta} e^{-\lambda^{1 / \alpha}}\right|^{-b}<\infty
$$

As we now show, it is an exercise in calculus to see that the left side is bounded for $\alpha>b-1 \geqq 0$. 
Making the substitution $u=\lambda^{1 / \alpha}$ and writing the absolute value on the right side as the square root of the sum of the squares of its real and imaginary parts leads to an upper bound for (3.23) of the form

$$
\int_{0}^{1} d \theta \int_{0}^{1} d u u^{\alpha-1}\left[\left(1-e^{-u}\right)^{2}+e^{-2 u} \theta^{2}\right]^{-b / 2} .
$$

The change of variables $\theta_{1}=\theta e^{-u} /\left(1-e^{-u}\right)$ in (3.24) gives

$$
\int_{0}^{1} d u u^{\alpha-1} \frac{1-e^{-u}}{e^{-u}}\left(1-e^{-u}\right)^{-b} \int_{0}^{e^{-u /\left(1-e^{-u}\right)}} d \theta_{1}\left[1+\theta_{1}^{2}\right]^{-b / 2} .
$$

The $\theta_{1}$-integral is bounded uniformly in $u$ if $b>1$, while if $b=1$ it is finite for $u$ near 1 and $O(|\log u|)$ for $u$ near 0 . Hence for $b \geqq 1,(3.25)$ is bounded above by a multiple of

$$
\int_{0}^{1} d u u^{\alpha-b}|\log u|
$$

which is finite for $\alpha>b-1$.

(ii) Given the bound on the derivative, it follows from (i) that $\left|n a_{n}\right| \leqq O\left(R^{-n} n^{p}\right)$ for any $p>b-1$. Therefore $\left|a_{n}\right| \leqq O\left(R^{-n} n^{p-1}\right)$ for any $\alpha \equiv 1-p<2-b$.

Remark. The hypothesis $b \geqq 1$ in Lemma 3.3(i) is not artificial. For example, let $f(z)=\sum_{n=1}^{\infty} n^{-2} z^{2^{n}}$. Then $f(z)$ is finite for $|z| \leqq 1$ so in particular $|f(z)| \leqq$ const. $|1-z|^{-b}$ for any $b \in[0,1)$. However $a_{N}=\left[\log _{2} N\right]^{-2}$ for $N=2^{n}$, so $a_{n} \neq O\left(n^{b-1+\varepsilon}\right)$ for $\varepsilon \in(0,1-b)$.

The following lemma is a kind of Tauberian theorem, in which information more detailed than merely the asymptotic form of a power series near its singularity provides information about the large- $n$ asymptotics of the coefficients of the power series.

Lemma 3.4. Let

$$
f(z)=\frac{1}{R-z-\varphi(z)}=\sum_{n=0}^{\infty} b_{n} z^{n},
$$

where $\varphi(z)=\sum_{n=0}^{\infty} a_{n} z^{n}$. Suppose that for some $\varepsilon \in(0,1), \sum_{n=0}^{\infty} n^{1+\varepsilon}\left|a_{n}\right| R^{n}<\infty$, so in particular $\varphi(z)$ and $\varphi^{\prime}(z)$ are both finite for $|z|=R$. Assume in addition that $\varphi^{\prime}(R) \neq-1$. Suppose that $\varphi(R)=0$ but that $R-z-\varphi(z) \neq 0$ for $|z| \leqq R, z \neq R$. Then

$$
f(z)=\frac{1}{1+\varphi^{\prime}(R)} \frac{1}{R-z}+O\left(|R-z|^{\varepsilon-1}\right)
$$

uniformly in $|z| \leqq R$, and

$$
b_{n}=R^{-n-1}\left[\frac{1}{1+\varphi^{\prime}(R)}+O\left(n^{-\alpha}\right)\right] \text { as } n \rightarrow \infty,
$$

for every $\alpha<\varepsilon$. 
Proof. Since $\varphi(R)=0$,

$$
\begin{aligned}
f(z) & =\frac{1}{R-z+\varphi(R)-\varphi(z)} \\
& =\frac{1}{R-z+\varphi^{\prime}(R)(R-z)+\left[\varphi(R)-\varphi(z)-\varphi^{\prime}(R)(R-z)\right]} .
\end{aligned}
$$

Let

$$
h(z)=\frac{\varphi(R)-\varphi(z)-\varphi^{\prime}(R)(R-z)}{R-z}
$$

and

$$
\psi(z)=-\frac{h(z)}{1+\varphi^{\prime}(R)+h(z)}
$$

A little algebra gives

$$
f(z)=\frac{1}{1+\varphi^{\prime}(R)} \frac{1}{R-z}[1+\psi(z)] .
$$

Since $|h(z)| \leqq O\left(|R-z|^{\varepsilon}\right)$ uniformly in $|z| \leqq R$ by Lemma 3.2 , it is also the case that $|\psi(z)| \leqq O\left(|R-z|^{\varepsilon}\right)$ uniformly in $|z| \leqq R$. This proves (3.27).

Let $C_{r}$ be the circle of radius $r$ centred at the origin and oriented counterclockwise. The coefficient $b_{n}$ is given by the contour integral

$$
b_{n}=\frac{1}{2 \pi i} \int_{C_{R / 2}} \frac{f(z)}{z^{n+1}} d z
$$

so by (3.32)

$$
b_{n}=\frac{1}{1+\varphi^{\prime}(R)} \frac{1}{R^{n+1}}+\frac{1}{1+\varphi^{\prime}(R)} \frac{1}{2 \pi i} \oint_{C_{R / 2}} \frac{\psi(z)}{(R-z) z^{n+1}} d z .
$$

It remains to show that the second term gives a correction of the desired size.

We use statement (ii) of Lemma 3.3 for the correction term, as follows. A straightforward calculation using the assumed bound on the $(1+\varepsilon)$-derivative of $\varphi$, together with Lemma 3.2, gives

$$
\left|\frac{d}{d z} \frac{\psi(z)}{R-z}\right| \leqq \text { const. }|R-z|^{\varepsilon-2}
$$

uniformly in $|z| \leqq R$. Hence the coefficient of $z^{n}$ of $(R-z)^{-1} \psi(z)$ is bounded above by $O\left(R^{-n} n^{-\alpha}\right)$, for every $\alpha<\varepsilon$, by Lemma 3.3(ii). This gives the required bound on the second term of (3.34).

3.3. Fractional Derivatives of the Propagator. In this section we begin by obtaining bounds on norms of fractional derivatives of the propagator. These are then used in conjunction with Lemma 2.4 to obtain bounds on fractional derivatives of $\hat{\Pi}_{z}(k)$. In a final lemma, a bound on $\hat{G}_{z}(0)$ is obtained. 
For $\lambda \geqq 0$ we define

$$
p_{\lambda}=z_{c} e^{-\lambda^{1 /(1-\varepsilon)}} .
$$

We also write

$$
\hat{F}_{z}(k)=\frac{1}{\hat{G}_{z}(k)}=1-2 d z \hat{D}(k)-\hat{\Pi}_{z}(k) .
$$

The following two lemmas will be used to bound fractional derivatives of the propagator.

Lemma 3.5. Let $d \geqq 5$. There is a positive constant $c$ such that for any $k$ and any $\lambda$

$$
\hat{F}_{p_{\lambda}}(k) \geqq c z_{c}\left[1-e^{-\lambda^{1 /(1-\varepsilon)}} \hat{D}(k)\right] .
$$

Proof. Since $\hat{F}_{z_{c}}(0)=0$,

$$
\begin{aligned}
\hat{F}_{p_{\lambda}}(k) & =\left[\hat{F}_{p_{\lambda}}(k)-\hat{F}_{p_{\lambda}}(0)\right]+\left[\hat{F}_{p_{\lambda}}(0)-\hat{F}_{z_{c}}(0)\right] \\
& =2 d p_{\lambda}[1-\hat{D}(k)]+\left[\hat{\Pi}_{p_{\lambda}}(0)-\hat{\Pi}_{p_{\lambda}}(k)\right]+\int_{p_{\lambda}}^{z_{c}}\left[-\partial_{p} \hat{F}_{p}(0)\right] d p .
\end{aligned}
$$

By Theorem 2.8, there are positive constants $C_{3}$ and $\lambda_{0}$ such that for $\lambda \leqq \lambda_{0}$ and $p \in\left[p_{\lambda}, z_{c}\right]$,

$$
-\partial_{p} \hat{F}_{p}(0) \geqq C_{3}>0
$$

Therefore for $\lambda \leqq \lambda_{0}$,

$$
\int_{p_{\lambda}}^{z_{c}}\left[-\partial_{p} \hat{F}_{p}(0)\right] d p \geqq C_{3}\left(z_{c}-p_{\lambda}\right) .
$$

By Theorem 2.8, $\quad \hat{\Pi}_{p_{\lambda}}(0)-\hat{\Pi}_{p_{\lambda}}(k) \geqq-C_{5}[1-\hat{D}(k)] . \quad$ Let $\quad c=\min \{2 d$ $\left.-C_{5} p_{\lambda_{0}}^{-1}, C_{3}\right\}$. Then by Theorem $2.8, c>0$ (decreasing $\lambda_{0}$ if necessary), and for $\lambda \leqq \lambda_{0}$ we have the desired bound

$$
\begin{aligned}
\hat{F}_{p_{\lambda}}(k) & \geqq\left(2 d-C_{5} p_{\lambda}^{-1}\right) p_{\lambda}[1-\hat{D}(k)]+C_{3}\left(z_{c}-p_{\lambda}\right) \\
& \geqq c p_{\lambda}[1-\hat{D}(k)]+c\left(z_{c}-p_{\lambda}\right) \\
& =c z_{c}\left[1-e^{-\lambda^{1 /(1-\varepsilon)}} \hat{D}(k)\right] .
\end{aligned}
$$

But for $\lambda \geqq \lambda_{0}$,

$$
\hat{G}_{p_{\lambda}}(k) \leqq \hat{G}_{p_{\lambda}}(0) \leqq\left.\hat{G}_{z}(0)\right|_{z=z_{c} e^{-\lambda_{0}^{1 /(1-\varepsilon)}}}<\infty .
$$

Hence for $\lambda \geqq \lambda_{0} \hat{F}_{p_{\lambda}}(k)$ is bounded below by a positive constant, and so (3.38) holds (decreasing $c$ if necessary).

For the next lemma, we define

$$
H_{z}(x)=z^{-1}\left[G_{z}(x)-\delta_{0, x}\right] .
$$

Lemma 3.6. For any $p \in\left(0, z_{c}\right]$ and $m=1,2,3, \ldots$,

$$
\partial_{p}^{m} G_{p}(x) \leqq m ! H_{p} * \ldots * H_{p} * G_{p}(x),
$$

where there are $m$ factors of $H_{p}$ in the convolution. 
Proof. By definition,

$$
\partial_{p}^{m} G_{p}(x)=p^{-m} m ! \sum_{\omega: 0 \rightarrow x}\left(\begin{array}{c}
|\omega| \\
m
\end{array}\right) p^{|\omega|}
$$

where the sum is over all self-avoiding walks from 0 to $x$. The binomial coefficient on the right side counts the number of ways of choosing $0<i_{1}<i_{2}<\ldots$ $<i_{m} \leqq|\omega|$, so it is also the number of ways to break $\omega$ into $m+1$ pieces such that the first $m$ pieces each consist of at least one step. The upper bound then follows by neglecting the mutual avoidance between these pieces.

We are now able to obtain bounds on fractional derivatives of the propagator. For this we introduce the notation $i(d)$ to denote the integer $(d-4) / 2$ if $d$ is even, and $(d-3) / 2$ if $d$ is odd. The bound (3.47) in the statement of the next theorem proves Theorem 1.3, and should be optimal in the sense that $(d-2) / 2$ or more derivatives would diverge (as they do for simple random walk). For $d>5$ the bounds (3.48) and (3.49) can be improved, but for simplicity we state only the results valid for all $d \geqq 5$.

Theorem 3.7. Let $d \geqq 5$. There are positive (dimension-dependent) constants $K_{1}(\varepsilon)$, $K_{2}(\varepsilon)$ and $K_{3}(\varepsilon)$ such that for any $p \in\left(0, z_{c}\right]$,

$$
\begin{gathered}
\left\|\delta_{p}^{\varepsilon} \partial_{p}^{i(d)} G_{p}(x)\right\|_{\infty} \leqq K_{1}(\varepsilon) \quad \text { if } \varepsilon \in\left(0, \frac{d-2}{2}-i(d)\right), \\
\left\|\delta_{p}^{\varepsilon} G_{p}(x)\right\|_{2} \leqq K_{2}(\varepsilon) \quad \text { if } \varepsilon \in(0,1 / 4)
\end{gathered}
$$

and

$$
\left\||x|^{2} \delta_{p}^{\varepsilon} G_{p}(x)\right\|_{\infty} \leqq K_{3}(\varepsilon) \quad \text { if } \varepsilon \in(0,1 / 2) .
$$

Proof. For an upper bound, we take $p=z_{c}$. We define $p_{\lambda}$ as in (3.36). The proof of each of these three inequalities is similar, and we focus mainly on the first one. By Lemma 3.1 [using the fact that $G_{p}(x)$ has nonnegative coefficients $c_{n}(x)$ ], we have

$$
\delta_{p}^{\varepsilon} \partial_{p}^{i(d)} G_{p}(x)=\left.C_{1-\varepsilon} z_{c} \int_{0}^{\infty} \partial_{p}^{i(d)+1} G_{p}(x)\right|_{p=p_{\lambda}} e^{-\lambda^{1 /(1-\varepsilon)}} d \lambda .
$$

Using Lemma 3.6 and the Fourier transform we can bound the right side as

$$
\delta_{p}^{\varepsilon} \partial_{p}^{i(d)} G_{p}(x) \leqq[i(d)+1] ! \int \frac{d^{d} k}{(2 \pi)^{d}} C_{1-\varepsilon} z_{c} \int_{0}^{\infty} d \lambda e^{-\lambda^{1 /(1-\varepsilon)}}\left|\hat{H}_{p_{\lambda}}(k)^{i(d)+1} \hat{G}_{p_{\lambda}}(k)\right| .
$$

It is not difficult to check (using Theorem 2.6) that there is a constant $K_{4}$, not depending on $\lambda$, such that

$$
\left|\hat{H}_{p_{\lambda}}(k)\right| \leqq K_{4} \hat{F}_{p_{\lambda}}(k)^{-1} .
$$

We bound the right side of (3.51), using (3.52) and (3.38), by

$$
\begin{aligned}
{[i(d)+1] ! K_{4}^{i(d)+1} \int \frac{d^{d} k}{(2 \pi)^{d}} C_{1-\varepsilon} z_{c} \int_{0}^{\infty} d \lambda e^{-\lambda^{1 /(1-\varepsilon)}} \hat{F}_{p_{\lambda}}(k)^{-(i(d)+2)} } \\
\leqq \text { const. } \int \frac{d^{d} k}{(2 \pi)^{d}} \int_{0}^{\infty} d \lambda e^{-\lambda^{1 /(1-\varepsilon)}}\left[1-e^{-\lambda^{1 /(1-\varepsilon)}} \hat{D}(k)\right]^{-(i(d)+2)} .
\end{aligned}
$$


Now by (3.9), the right side of the above inequality is equal to

$$
\begin{aligned}
& \text { const. }\left.\int \frac{d^{d} k}{(2 \pi)^{d}} \frac{1}{\hat{D}(k)} \delta_{p}^{\varepsilon}\left[(1-p \hat{D}(k))^{-i(d)-1}\right]\right|_{p=1} \\
= & \text { const. } \int \frac{d^{d} k}{(2 \pi)^{d}} \sum_{n=i(d)+1}^{\infty} n(n-1) \ldots(n-i(d)+1)(n-i(d))^{\varepsilon} \hat{D}(k)^{n-i(d)-1} .
\end{aligned}
$$

Since

$$
\int\left|\hat{D}(k)^{n}\right| \frac{d^{d} k}{(2 \pi)^{d}} \leqq O\left(n^{-d / 2}\right),
$$

the right side is finite for $i(d)+\varepsilon-(d / 2)<-1$, i.e., $\varepsilon<(d-2) / 2-i(d)$.

For (3.48), we proceed in a similar fashion. Using Lemmas 3.1 and 3.6 and the Parseval relation gives

$$
\left\|\delta_{p}^{\varepsilon} G_{p}(x)\right\|_{2} \leqq\left\|C_{1-\varepsilon} z_{c} \int_{0}^{\infty} d \lambda e^{-\lambda^{1 /(1-\varepsilon)}} \hat{H}_{p_{\lambda}}(k) \hat{G}_{p_{\lambda}}(k)\right\|_{2},
$$

where

$$
\|\hat{g}(k)\|_{2} \equiv\left[\int \hat{g}(k)^{2} \frac{d^{d} k}{(2 \pi)^{d}}\right]^{1 / 2} .
$$

Arguing as above and using the triangle inequality for $\|\cdot\|_{2}$ gives

$$
\left\|\delta_{p}^{\varepsilon} G_{p}(x)\right\|_{2} \leqq z_{c}^{-1} c^{-2} K_{4} \sum_{n=1}^{\infty} n^{\varepsilon}\left\|\hat{D}(k)^{n-1}\right\|_{2} .
$$

The desired bound now follows from the fact that $\left\|\hat{D}(k)^{n}\right\|_{2} \leqq O\left(n^{-d / 4}\right)$.

For (3.49), we again use Lemmas 3.1 and 3.6 and the Fourier transform, with the result

$$
|x|^{2} \delta_{p}^{\varepsilon} G_{p}(x) \leqq C_{1-\varepsilon} z_{c} \int_{0}^{\infty} d \lambda e^{-\lambda^{1 /(1-\varepsilon)}} \int \frac{d^{d} k}{(2 \pi)^{d}} \sum_{\mu=1}^{d}\left|\partial_{\mu}^{2}\left[\hat{H}_{p_{\lambda}}(k) \hat{G}_{p_{\lambda}}(k)\right]\right| .
$$

By Theorem 2.6, $\left|\partial_{\mu}^{2} \hat{F}_{p_{\lambda}}(k)\right|$ is bounded. Also, it follows from Taylor's theorem and the bound on $\left|\partial_{\mu} \hat{\Pi}_{z}(k)\right|$ of Theorem 2.6 that

$$
\sum_{\mu=1}^{d}\left[\partial_{\mu} \hat{F}_{p_{\lambda}}(k)\right]^{2} \leqq \text { const. } k^{2} \leqq \text { const. }\left[1-e^{-\lambda^{1 /(1-\varepsilon)}} \hat{D}(k)\right] .
$$

It then follows from direct computation of the second derivative in (3.59) that for some constant $K_{5}$,

$$
|x|^{2} \delta_{p}^{\varepsilon} G_{p}(x) \leqq C_{1-\varepsilon} z_{c} \int_{0}^{\infty} d \lambda e^{-\lambda^{1 /(1-\varepsilon)}} \int \frac{d^{d} k}{(2 \pi)^{d}} K_{5} \hat{F}_{p_{\lambda}}(k)^{-3} .
$$

Now the argument proceeds as below (3.52).

The following result follows immediately from Lemma 2.4 , Theorem 2.5 , and the above theorem. (In fact, the $\varepsilon=0$ version of (3.49) given in Theorem 2.5 is sufficient for Corollary 3.8. The $\varepsilon>0$ version is used in Sects. 3.5 and 3.6.) 
Corollary 3.8. Let $d \geqq 5$. There is a finite positive $K_{6}(\varepsilon)$ such that for any $k \in$ $[-\pi, \pi]^{d}$ and $|z| \leqq z_{c}$,

$$
\left|\delta_{z}^{\varepsilon} \partial_{z} \hat{\Pi}_{z}(k)\right|,\left|\delta_{z}^{\varepsilon} \partial_{\mu}^{u} \hat{\Pi}_{z}(k)\right| \leqq K_{6}(\varepsilon),
$$

for $u=0,1,2$, where the first bound holds for any positive $\varepsilon<1 / 2$ and the second for any positive $\varepsilon<1 / 4$. In fact the series representations of the left side are bounded absolutely by $K_{6}(\varepsilon)$.

We end this section with an upper bound on the zero-momentum propagator, for $z$ in the closed disk of radius $z_{c}$.

Lemma 3.9. Let $d \geqq 5$. There is a constant $c>0$ such that for any $z$ with $|z| \leqq z_{c}$, $\hat{F}_{z}(0) \geqq c\left|z_{c}-z\right|$.

Proof. By definition of $z_{c}, \hat{F}_{z}(0)$ is nonzero for $|z|<z_{c}$. By Theorem $2.7, \hat{F}_{z}(0)$ is also nonzero on the circle of convergence $|z|=z_{c}$, except at $z=z_{c}$. Since $\hat{F}_{z}(0)$ is continuous in $z$ for $|z| \leqq z_{c}$, it thus suffices to obtain the inequality in the statement of the lemma for $z$ in a neighbourhood of $z_{c}$.

By Corollary 3.8, we have an absolute uniform bound on $\left|\delta_{z}^{\varepsilon} \partial_{z} \hat{F}_{z}(k)\right|$ for $|z| \leqq z_{c}$. Therefore, by Lemma 3.2 there is a constant $B$ such that

$$
\left|\hat{F}_{z}(0)-\partial_{z} \hat{F}_{z_{c}}(0)\left(z-z_{c}\right)\right| \leqq B\left|z_{c}-z\right|^{1+\varepsilon}
$$

for all $|z| \leqq z_{c}$. Since (by Theorem 2.8 ) $-\partial_{z} \hat{F}_{z_{c}}(0) \geqq C_{3}>0$, it follows that there are positive constants $c, \delta_{0}$ such that

$$
\left|\hat{F}_{z}(0)\right| \geqq c\left|z_{c}-z\right|
$$

for $z \in\left\{|z| \leqq z_{c}\right\} \cap\left\{\left|z_{c}-z\right|<\delta_{0}\right\}$.

3.4. Proof of Theorem 1.1. In this section we give the proof of Theorem 1.1. (Bounds on $A$ and $D$ for $d=5$ are discussed at the end of Sect. II.1.3.) We begin with $c_{n}$.

Proof of Theorem 1.1(a). Since $\hat{F}_{z_{c}}(0)=0$, the susceptibility can be written as

$$
\chi(z)=\frac{1}{2 d}\left[\frac{1}{z_{c}-z-\varphi(z)}\right],
$$

where

$$
\varphi(z)=\frac{1}{2 d}\left[\hat{\Pi}_{z}(0)-\hat{\Pi}_{z_{c}}(0)\right]
$$

By Corollary 3.8, for any $\varepsilon<1 / 2, \sum_{n} n^{1+\varepsilon}\left|\pi_{n}\right| z_{c}^{n}<\infty$, where $\pi_{n}$ is the coefficient of $z^{n}$ in the power series representation of $\hat{\Pi}_{z}(0)$. Moreover by Theorem 2.8 $\varphi^{\prime}\left(z_{c}\right) \neq-1$, and by Theorem 2.7 the only zero of $\varphi(z)$ on the circle $|z|=z_{c}$ is at $z=z_{c}$. It then follows immediately from Lemma 3.4 that

$$
\begin{aligned}
c_{n} & =\frac{1}{2 d} z_{c}^{-n-1}\left[\frac{1}{1+\frac{1}{2 d} \partial_{z} \hat{\Pi}_{z_{c}}(0)}+O\left(n^{-\varepsilon}\right)\right] \\
& =A \mu^{n}\left[1+O\left(n^{-\varepsilon}\right)\right],
\end{aligned}
$$

where $A$ is given by (3.3). 
We now turn to the mean square displacement.

Proof of Theorem 1.1(b). By definition of the Fourier transform,

$$
\left\langle|\omega(n)|^{2}\right\rangle_{n}=\frac{-\nabla_{k}^{2} \hat{c}_{n}(0)}{c_{n}} .
$$

The asymptotic behaviour of the denominator on the right side was obtained in (3.67), and we now proceed to analyze the numerator.

Since $\hat{c}_{n}(k)$ is the coefficient of $z^{n}$ in $\hat{G}_{z}(k)$,

$$
-\nabla_{k}^{2} \hat{c}_{n}(0)=-\frac{1}{2 \pi i} \oint \nabla_{k}^{2} \hat{G}_{z}(0) \frac{d z}{z^{n+1}}=\frac{1}{2 \pi i} \oint \frac{\nabla_{k}^{2} \hat{F}_{z}(0)}{\hat{F}_{z}(0)^{2}} \frac{d z}{z^{n+1}},
$$

where the integrals are performed around a small circle centred at the origin. We define an error term $E(z)$ by

$$
\frac{\nabla_{k}^{2} \hat{F}_{z}(0)}{\hat{F}_{z}(0)^{2}}=\frac{\nabla_{k}^{2} \hat{F}_{z_{c}}(0)}{\left[\partial_{z} \hat{F}_{z_{c}}(0)\right]^{2}\left(z_{c}-z\right)^{2}}+E(z) .
$$

Inserting the right side of (3.70) into the right side of (3.69), the integral corresponding to the first term can be performed exactly to give

$$
-\nabla_{k}^{2} \hat{c}_{n}(0)=\frac{\nabla_{k}^{2} \hat{F}_{z_{c}}(0)}{\left[\partial_{z} \hat{F}_{z_{c}}(0)\right]^{2}}(n+1) z_{c}^{-(n+2)}+\frac{1}{2 \pi i} \oint E(z) \frac{d z}{z^{n+1}} .
$$

The remaining task is to bound the last term in (3.71). This is done using Lemma 3.3. In fact, it follows from Lemma 3.3(i) that if it can be shown that for every $\varepsilon<1 / 4,|E(z)| \leqq$ const. $\left|z_{c}-z\right|^{-2+\varepsilon}$ for all $|z| \leqq z_{c}$, then the second term on the right side of (3.71) is $O\left(z_{c}^{-n} n^{\alpha}\right)$ for every $\alpha>3 / 4$. Assuming for the moment this bound on the error term and using (3.67), we then have the desired result

$$
\left\langle|\omega(n)|^{2}\right\rangle_{n}=D n+O\left(n^{\alpha}\right),
$$

with $D$ given by (3.5).

We now establish the upper bound on $|E(z)|$ used in the previous paragraph. We first use (3.70) to write $E(z)$ as a difference of two fractions, and then write this difference over a common denominator and add and subtract $\nabla_{k}^{2} \hat{F}_{z}(0) \hat{F}_{z}(0)^{2}$ in the numerator. This leads to

with

$$
E(z)=T_{1}+T_{2}
$$

$$
T_{1}=\left[\partial_{z} \hat{F}_{z_{c}}(0)\right]^{-2} \frac{\nabla_{k}^{2} \hat{F}_{z}(0)-\nabla_{k}^{2} \hat{F}_{z_{c}}(0)}{\left(z_{c}-z\right)^{2}}
$$

and

$$
T_{2}=\frac{-\nabla_{k}^{2} \hat{F}_{z}(0)\left[\hat{F}_{z}(0)^{2}-\left[\partial_{z} \hat{F}_{z_{c}}(0)\right]^{2}\left(z_{c}-z\right)^{2}\right]}{\left[\partial_{z} \hat{F}_{z_{c}}(0)\right]^{2} \hat{F}_{z}(0)^{2}\left(z_{c}-z\right)^{2}} .
$$

Fix $\varepsilon<1 / 4$. For $T_{1}$, we use existence of an $\varepsilon$-derivative in the numerator by Corollary 3.8, together with the Taylor theorem type bound of (3.14) to conclude that

$$
\left|T_{1}\right| \leqq O\left(\left|z_{c}-z\right|^{\varepsilon-2}\right) .
$$

For $T_{2}$ we factor the difference of squares in the numerator and use Lemma 3.9 to 
bound the denominator, obtaining

$$
\left|T_{2}\right| \leqq \text { const. }\left(z_{c}-z\right)^{-4}\left[\hat{F}_{z}(0)+\partial_{z} \hat{F}_{z_{c}}(0)\left(z_{c}-z\right)\right]\left[\hat{F}_{z}(0)-\partial_{z} \hat{F}_{z_{c}}(0)\left(z_{c}-z\right)\right] .
$$

The middle factor on the right side is $O\left(\left|z_{c}-z\right|^{1+\varepsilon}\right)$, by (3.63). For the last factor, the second term is clearly $O\left(\left|z_{c}-z\right|\right)$, as is the first, again by (3.63). Therefore $\left|T_{2}\right| \leqq O\left(\left|z_{c}-z\right|^{\varepsilon-2}\right)$.

3.5. Proof of Theorem 1.4. In this section we prove Theorem 1.4. The proof basically follows the method used in [15] to study the analogous problem for percolation, but differs in its use of fractional derivatives. The use of fractional derivatives simplifies some aspects of the proof, and gives the stronger result of asymptotic behaviour of $m(p)$, rather than upper and lower bounds.

We work in this section only with positive activity $p<z_{c}$, and denote the inverse of the correlation length (1.8) by $m(p)$ :

$$
m(p)=-\lim _{n \rightarrow \infty} n^{-1} \log G_{p}\left(n e_{1}\right) .
$$

It was shown in [9] that $m(p) \searrow 0$ as $p \nearrow z_{c}$.

For any function $f$ defined on $\mathbf{Z}^{d}$, and $m \in \mathbf{R}$, we define

$$
f^{(m)}(x)=f(x) e^{m x_{1}} .
$$

For $m<m(p)$, let $\chi^{(m)}(p)=\sum_{x} G_{p}^{(m)}(x)$. As usual $B(p)=\sum_{x \neq 0} G_{p}(x)^{2}$, and we let $B^{(m)}(p)=\sum_{x \neq 0} G_{p}^{(m)}(x)^{2}$.

By multiplying (2.11) by $e^{m x_{1}}$ and then taking the Fourier transform, we obtain

$$
\hat{G}_{p}^{(m)}(k)=\frac{1}{1-2 d p \hat{D}^{(m)}(k)-\hat{\Pi}_{p}^{(m)}(k)} .
$$

It is not clear a priori that the Fourier transform $\hat{\Pi}_{p}^{(m)}$ exists, but we will show in the following that it does, for $p<z_{c}$ and $m<m(p)$. By definition,

$$
\hat{D}^{(m)}(k)=\frac{1}{2 d} \sum_{y:|y|=1} e^{m y_{1}} e^{i k \cdot y},
$$

and hence

$$
\hat{D}^{(m)}(0)=\frac{1}{d}[\cosh m+d-1] .
$$

Therefore

$$
\chi(p)^{-1}-\chi^{(m)}(p)^{-1}=2 p[\cosh m-1]+\hat{\Pi}_{p}^{(m)}(0)-\hat{\Pi}_{p}(0) .
$$

We intend to take the limit as $m \nearrow m(p)$ in (3.83). Arguing as in the proof of Corollary A.4 of [15], it can be seen that $\chi^{(m(p))}(p)=\infty$. Since results of [9] imply that $\chi^{(m)}(p)$ is finite for $m<m(p)$, it follows from the monotone convergence theorem that for any $p<z_{c}$,

$$
\lim _{m>m(p)} \chi^{(m)}(p)=\infty
$$

To understand the behaviour of the last two terms on the right side of (3.83) as $m \nearrow m(p)$, we proceed as follows. 
By definition and symmetry,

$$
\begin{aligned}
\hat{\Pi}_{p}^{(m)}(0)-\hat{\Pi}_{p}(0) & =\sum_{x}\left[\cosh m x_{1}-1\right] \Pi_{p}(x) \\
& =\frac{m^{2}}{2 d} \sum_{x}|x|^{2} \Pi_{p}(x)+\sum_{x}\left[\cosh m x_{1}-1-\frac{m^{2} x_{1}^{2}}{2}\right] \Pi_{p}(x) .
\end{aligned}
$$

There is a positive constant $C$ such that for any $\varepsilon \in[0,2]$,

$$
0 \leqq \cosh m x_{1}-1-\frac{m^{2} x_{1}^{2}}{2} \leqq C m^{2+\varepsilon}\left|x_{1}\right|^{2+\varepsilon} \cosh m x_{1}
$$

Therefore

$$
\left|\sum_{x}\left[\cosh m x_{1}-1-\frac{m^{2} x_{1}^{2}}{2}\right] \Pi_{p}(x)\right| \leqq C m^{2+\varepsilon} \sum_{x}\left|x_{1}\right|^{2+\varepsilon}\left|\Pi_{p}^{(m)}(x)\right| .
$$

Suppose we knew that the sum on the right side of this inequality was $O(1)$ uniformly in $m<m(p)$ and $p<z_{c}$. [In particular this would imply existence of $\hat{\Pi}_{p}^{(m)}(k)$.] Then the limit of the left side, as $m \nearrow m(p)$ would be $O\left(m(p)^{2+\varepsilon}\right)$. Assuming this, and using (3.84) and (3.85), taking the limit as $m \nearrow m(p)$ in (3.83) gives

$$
\chi(p)^{-1}=2 p[\cosh m(p)-1]+\frac{m(p)^{2}}{2 d} \sum_{x}|x|^{2} \Pi_{p}(x)+O\left(m(p)^{2+\varepsilon}\right) .
$$

As $p \nearrow z_{c}, m(p) \searrow 0$ and hence the right side of (3.88) is asymptotic to

$$
m(p)^{2}\left[z_{c}+\frac{1}{2 d} \sum_{x}|x|^{2} \Pi_{z_{c}}(x)\right] .
$$

The quantity in square brackets is positive by Theorem 2.8 . Also, by Theorem 1.2 the left side of $(3.88)$ is asymptotic to $\left(A z_{c}\right)^{-1}\left(z_{c}-p\right)$. Therefore

$$
m(p)^{2} \sim \frac{2 d}{A\left[2 d z_{c}+\sum_{x}|x|^{2} \Pi_{z_{c}}(x)\right]} \frac{z_{c}-p}{z_{c}},
$$

and Theorem 1.4 would be proved [using (3.3) and (3.5)], once we show the sum over $x$ on the right side of (3.87) is $O(1)$.

We now show that the sum over $x$ on the right side of (3.87) is uniformly bounded, for $\varepsilon<1 / 2$. This sum involves diagrams having two or more loops, weighted with both $\left|x_{1}\right|^{2+\varepsilon}$ and $e^{m x_{1}}$. We split the former among subwalks along the lower side of the diagram using Hölder's inequality, and factor the latter along the upper side of the diagram. Lemma 2.3 is used to bound the diagrams. The subwalk weighted with $\left|x_{1}\right|^{2+\varepsilon}$ is bounded using the infinity norm, as follows:

$$
\begin{aligned}
\sup _{x}\left(\left|x_{1}\right|^{2+\varepsilon} \sum_{n} c_{n}(x) p^{n}\right) & \leqq \sup _{x}\left(\left|x_{1}\right|^{2} \sum_{n} n^{\varepsilon} c_{n}(x) p^{n}\right) \\
& =\sup _{x}\left(\left|x_{1}\right|^{2} \delta_{p}^{\varepsilon} G_{p}(x)\right) .
\end{aligned}
$$

The right side is finite, by Theorem 3.7. All other subwalks are bounded using the $L^{2}$ norm, yielding factors of $B(p)^{1 / 2},[1+B(p)]^{1 / 2}, B^{(m)}(p)^{1 / 2}$ and $\left[1+B^{(m)}(p)\right]^{1 / 2}$. The sum of all diagrams is then bounded above by a geometric series with an $m$-dependent ratio. If we knew that for some $\delta>0, B^{(m)}(p)$ were bounded uniformly 
in $m<m(p)$ and $p \in\left[z_{c}-\delta, z_{c}\right)$ by a constant marginally larger than $B\left(z_{c}\right)$, the geometric series would converge and the proof would be complete. This last step is completed in the following lemma.

Lemma 3.10. For any $\gamma>0$ (sufficiently small), there is a $\delta>0$ such that for $p \in\left[z_{c}-\delta, z_{c}\right]$ and $m<m(p)$,

$$
B^{(m)}(p)<B\left(z_{c}\right)+\gamma .
$$

Proof. Let $\gamma>0$ and $\varepsilon<1 / 2$. For any $p<z_{c}, B^{(m)}(p)$ is continuous in $m \in[0, m(p))$. This follows from the monotone convergence theorem and the fact that for $p<z_{c}$ and $m<m(p), B^{(m)}(p)<\infty$. (The finiteness of $B^{(m)}(p)$ follows from the fact [9] that $G_{p}(x) \leqq$ const. $(p) \exp \left[-m(p)\|x\|_{\infty}\right]$.) Clearly $B^{(0)}(p)<B\left(z_{c}\right)$. Hence by the "forbidden region" argument of Lemma II.1.11 (now with $m$ playing the role of $p$ ) it suffices to show that there is a $\delta>0$ such that given $p \in\left[z_{c}-\delta, z_{c}\right]$ and $m<m(p)$, if $B^{(m)}(p)<B\left(z_{c}\right)+2 \gamma$ then in fact $B^{(m)}(p)<B\left(z_{c}\right)+\gamma$. The remainder of the proof is concerned with showing the existence of such a $\delta$.

Denoting the inverse of $\hat{G}_{p}^{(m)}(k)$ by $\hat{F}_{p}^{(m)}(k)$, we have

$$
\left|\hat{G}_{p}^{(m)}(k)\right| \leqq \frac{1}{\left|\operatorname{Re} \hat{F}_{p}^{(m)}(k)\right|} .
$$

Now

$$
\begin{aligned}
\operatorname{Re} \hat{F}_{p}^{(m)}(k)= & \chi^{(m)}(p)^{-1}+2 d p \operatorname{Re}\left[\hat{D}^{(m)}(0)-\hat{D}^{(m)}(k)\right] \\
& +\operatorname{Re}\left[\hat{\Pi}_{p}^{(m)}(0)-\hat{\Pi}_{p}^{(m)}(k)\right] \\
\geqq & 2 d p \operatorname{Re}\left[\hat{D}^{(m)}(0)-\hat{D}^{(m)}(k)\right]+\operatorname{Re}\left[\hat{\Pi}_{p}^{(m)}(0)-\hat{\Pi}_{p}^{(m)}(k)\right] .
\end{aligned}
$$

But

$$
\begin{aligned}
2 d p \operatorname{Re}\left[\hat{D}^{(m)}(0)-\hat{D}^{(m)}(k)\right] & =p \sum_{y:|y|=1} e^{m y_{1}}[1-\cos k \cdot y] \\
& =p \sum_{y:|y|=1} \cosh m y_{1}[1-\cos k \cdot y] \\
& \geqq 2 d p[1-\hat{D}(k)] .
\end{aligned}
$$

Also,

$$
\begin{aligned}
\operatorname{Re}\left[\hat{\Pi}_{p}^{(m)}(0)-\hat{\Pi}_{p}^{(m)}(k)\right] & \\
= & {\left[\hat{\Pi}_{p}(0)-\hat{\Pi}_{p}(k)\right]+\operatorname{Re}\left[\left(\hat{\Pi}_{p}^{(m)}(0)-\hat{\Pi}_{p}^{(m)}(k)\right)-\left(\hat{\Pi}_{p}(0)-\hat{\Pi}_{p}(k)\right)\right] } \\
& =\left[\hat{\Pi}_{p}(0)-\hat{\Pi}_{p}(k)\right]+\sum_{x}\left[\cosh m x_{1}-1\right] \hat{\Pi}_{p}(x)[1-\cos k \cdot x] .
\end{aligned}
$$

Therefore

Since

$$
\begin{aligned}
\operatorname{Re} \hat{F}_{p}^{(m)}(k) \geqq & 2 d p[1-\hat{D}(k)]+\hat{\Pi}_{p}(0)-\hat{\Pi}_{p}(k) \\
& +\sum_{x}\left[\cosh m x_{1}-1\right] \Pi_{p}(x)[1-\cos k \cdot x] .
\end{aligned}
$$

$$
0 \leqq \cosh m x_{1}-1 \leqq \text { const. }\left(m\left|x_{1}\right|\right)^{\varepsilon} \cosh m x_{1}
$$


and

$$
0 \leqq 1-\cos k \cdot x \leqq \frac{(k \cdot x)^{2}}{2} \leqq \frac{\pi^{2} d}{4}|x|^{2}[1-\hat{D}(k)]
$$

we have

$$
\begin{aligned}
& \left|\sum_{x}\left[\cosh m x_{1}-1\right] \Pi_{p}(x)[1-\cos k \cdot x]\right| \\
& \leqq K m^{\varepsilon}[1-\hat{D}(k)] \sum_{x}|x|^{2} d\left|x_{1}\right|^{\varepsilon}\left|\Pi_{p}^{(m)}(x)\right|
\end{aligned}
$$

for some constant $K$. Now using the assumption that $B^{(m)}(p)<B\left(z_{c}\right)+2 \gamma$, we bound the sum on the right side of (3.99) using the method described above the statement of the lemma (for $\gamma$ sufficiently small). The result is

$$
\left|\hat{G}_{p}^{(m)}(k)\right| \leqq \frac{1}{2 d p[1-\hat{D}(k)]+\hat{\Pi}_{p}(0)-\hat{\Pi}_{p}(k)-O\left(m^{\varepsilon}\right)[1-\hat{D}(k)]},
$$

where the $O\left(m^{\varepsilon}\right)$ is uniform in $p<z_{c}$. We denote the right side of $(3.100)$ by $g_{p}^{(m)}(k)$.

By definition, $B^{(m)}(p)=\left\|\hat{G}_{p}^{(m)}(k)\right\|_{2}^{2}-1$. Hence by $(3.100)$ and the triangle inequality,

$$
B^{(m)}(p) \leqq\left[\left\|g_{p}^{(0)}(k)\right\|_{2}+\left\|g_{p}^{(0)}(k)-g_{p}^{(m)}(k)\right\|_{2}\right]^{2}-1 .
$$

For the second term in square brackets, we put the difference over a common denominator and use Theorem 2.8 to bound the resulting denominator from below. The result is that the second term in square brackets is $O\left(\mathrm{~m}^{\varepsilon}\right)$ uniformly in $p$ near $z_{c}$. Since $m<m(p)$ and $m(p) \searrow 0$ as $p \nearrow z_{c}$, such a term is $o(1)$ as $p \nearrow z_{c}$. This, the triangle inequality, and the fact that $g_{z_{c}}^{(0)}(k)=\hat{G}_{z_{c}}(k)$ give

$$
B^{(m)}(p) \leqq\left[\left\|\hat{G}_{z_{c}}(k)\right\|_{2}+\left\|g_{p}^{(0)}(k)-g_{z_{c}}^{(0)}(k)\right\|_{2}+o(1)\right]^{2}-1 .
$$

It now suffices to show that the second term in square brackets is $o(1)$ as $p \rightarrow z_{c}$.

The difference whose norm is being taken in the second term is

$$
\frac{2 d\left(z_{c}-p\right)[1-\hat{D}(k)]+\hat{\Pi}_{z_{c}}(0)-\hat{\Pi}_{p}(0)-\left[\hat{\Pi}_{z_{c}}(k)-\hat{\Pi}_{p}(k)\right]}{\left\{2 d p[1-\hat{D}(k)]+\hat{\Pi}_{p}(0)-\hat{\Pi}_{p}(k)\right\}\left\{2 d z_{c}[1-\hat{D}(k)]+\hat{\Pi}_{z_{c}}(0)-\hat{\Pi}_{z_{c}}(k)\right\}} .
$$

By Theorem 2.8 the denominator can be bounded below by a multiple of $[1-\hat{D}(k)]^{2}$, uniformly in $p$ near $z_{c}$. Hence the contribution to the norm due to the first term in the numerator is $O\left(z_{c}-p\right)$. For the remaining part of the numerator, we begin by rewriting it as

$$
\sum_{n, x} \pi_{n}(x)\left(z_{c}^{n}-p^{n}\right)[1-\cos k \cdot x],
$$

where $\pi_{n}(x)$ denotes the coefficient of $z^{n}$ in $\Pi_{z}(x)$. Since $0 \leqq 1-\cos k \cdot x$ $\leqq$ const. $|x|^{2}[1-\hat{D}(k)],(3.104)$ is bounded above in absolute value by

$$
\text { const. }[1-\hat{D}(k)] \sum_{n, x}|x|^{2}\left|\pi_{n}(x)\right|\left(z_{c}^{n}-p^{n}\right) \text {. }
$$

It suffices to observe that the right side is bounded by a multiple of $[1-\hat{D}(k)]\left(z_{c}-p\right)^{\varepsilon}$ by Corollary 3.8 and the first bound of Lemma 3.2. 
3.6. The Infrared Bound. In this section we complete the proof of Theorem 1.5 by showing that as $k \rightarrow 0$,

$$
\hat{G}_{z_{c}}(k)=\frac{C}{k^{2}+O\left(k^{2+\varepsilon}\right)}
$$

for any $\varepsilon<1 / 2$.

Since $\hat{G}_{z_{c}}(0)^{-1}=0$,

$$
\hat{G}_{z_{c}}(k)^{-1}=2 d z_{c}[1-\hat{D}(k)]+\hat{\Pi}_{z_{c}}(0)-\hat{\Pi}_{z_{c}}(k) .
$$

The last two terms on the right side can be written as

$$
\frac{k^{2}}{2 d} \sum_{x}|x|^{2} \Pi_{z_{c}}(x)+\sum_{x}\left[1-\cos k \cdot x-\frac{(k \cdot x)^{2}}{2}\right] \Pi_{z_{c}}(x) .
$$

The quantity in square brackets on the right side can be bounded above by $K k^{2+\varepsilon}|x|^{2+\varepsilon}$, for some constant $K$. The sum over $x$ can then be bounded as in (3.91). The result is

$$
\hat{G}_{z_{c}}(k)^{-1}=k^{2}\left[C^{-1}+O\left(k^{\varepsilon}\right)\right],
$$

where

$$
C^{-1}=z_{c}+\frac{1}{2 d} \sum_{x}|x|^{2} \Pi_{z_{c}}(x)
$$

\section{Convergence to Brownian Motion}

In this section we prove Theorem 1.6. It suffices to prove convergence of the finite dimensional distributions to Gaussian distributions, and tightness [4]. Tightness follows from Theorem 1.1(a) and (b) exactly as in [33], and we do not repeat the details here.

To obtain convergence of the finite dimensional distributions, we modify the argument of [34] to avoid the use of a memory. First, we need some notation. We fix $0=t_{0}<t_{1}<t_{2}<\ldots<t_{N} \leqq 1$. Let $\mathbf{k}=\left(k^{(1)}, \ldots, k^{(N)}\right)$, where each $k^{(i)} \in \mathbf{R}^{d}$, and similarly for $\mathbf{x}$. We define $\mathbf{k} \cdot \mathbf{x}=\sum_{i=1}^{N} k^{(i)} \cdot x^{(i)}$. Let $\mathbf{a}=\left(a_{1}, \ldots, a_{N}\right)$, with each $a_{i}$ a nonnegative integer, and let

$$
\Delta \omega(\mathbf{a})=\left(\omega\left(a_{1}\right), \omega\left(a_{2}\right)-\omega\left(a_{1}\right), \ldots, \omega\left(a_{N}\right)-\omega\left(a_{N-1}\right)\right) .
$$

We define

$$
M(\mathbf{k}, \mathbf{a})=\sum_{\omega:|\omega|=a_{N}} e^{i \mathbf{k} \cdot \Delta \omega(\mathbf{a})} K\left[0, a_{N}\right]
$$

where the sum over $\omega$ is a sum over simple random walks, and $K[a, b]$ was defined in (2.5). As explained in more detail in [34], to obtain convergence of the finite dimensional distributions it suffices to show that for $N=1,2,3, \ldots$,

$$
\lim _{n \rightarrow \infty} c_{n t_{N}}^{-1} M(\mathbf{k} / \sqrt{n}, n \mathbf{t})=\exp \left[-\frac{D}{2 d} \sum_{i=1}^{N}\left(k^{(i)}\right)^{2}\left(t_{i}-t_{i-1}\right)\right] .
$$

Here $D$ is the diffusion constant of (3.5), and the $n \mathbf{t}$ and $n t_{N}$ on the left should be 
interpreted as $\left(\left[n t_{1}\right], \ldots,\left[n t_{N}\right]\right)$ and $\left[n t_{n}\right]$ respectively. To simplify the notation we will continue to omit the square brackets denoting integer part.

We obtain (4.3) for $N=1$ in Sect. 4.1, and prove (4.3) for $N \geqq 2$ by induction on $N$ in Sect. 4.2.

4.1. The Scaling Limit of the Endpoint. In this section we prove (4.3), for $N=1$. In fact, a minor generalization of (4.3) will be needed to take the induction step, and we prove the generalization here.

Theorem 4.1. Fix $d \geqq 5$. Let $h_{n}$ be any fixed nonnegative sequence with $\lim _{n \rightarrow \infty} h_{n}=0$, and $g=\left\{g_{n}\right\}$ be any real sequence with $\left|g_{n}\right| \leqq h_{n}$ for all $n$. Let $T=t\left(1-g_{n}\right)$. Then for any $k \in \mathbf{R}^{d}$,

$$
\lim _{n \rightarrow \infty} \frac{\hat{c}_{n T}(k / \sqrt{n})}{c_{n T}}=\exp \left[-D k^{2} t / 2 d\right]
$$

uniformly in $g$.

Proof. Fix any $\varepsilon<1 / 4$. By (3.67), the denominator of (4.4) can be written

$$
c_{n T}=z_{c}^{-n T-1}\left\{\left[-\partial_{z} \hat{F}_{z_{c}}(0)\right]^{-1}+O\left(n^{-\varepsilon}\right)\right\} .
$$

The numerator of (4.4) is the coefficient of $z^{n T}$ in $\hat{G}_{z}(k / \sqrt{n})$, and hence is given by

$$
\hat{c}_{n T}(k / \sqrt{n})=\frac{1}{2 \pi i} \oint \frac{1}{\hat{F}_{z}(k / \sqrt{n})} \frac{d z}{z^{n T+1}},
$$

where the integration contour is a small circle centred at the origin. The task now is to obtain the asymptotic form of the integral on the right side.

We extract the leading contribution to the right side of (4.6) as follows. We subtract $\hat{F}_{z_{c}}(0)=0$ from $\hat{F}_{z}(k / \sqrt{n})$, and then add and subtract

$$
\partial_{z} \hat{\Pi}_{z_{c}}(0)\left(z_{c}-z\right)+\frac{1}{2 d} \nabla_{k}^{2} \hat{\Pi}_{z_{c}}(0) \frac{k^{2}}{n} .
$$

The result can be written

$$
\hat{F}_{z}(k / \sqrt{n})=\alpha-\beta z+E,
$$

where

and

$$
\begin{aligned}
& \alpha=\alpha(k / \sqrt{n})=-z_{c} \partial_{z} \hat{F}_{z_{c}}(0)-\frac{1}{2 d} \nabla_{k}^{2} \hat{\Pi}_{z_{c}}(0) \frac{k^{2}}{n}, \\
& \beta=\beta(k / \sqrt{n})=-\partial_{z} \hat{F}_{z_{c}}(0)-2 d[1-\hat{D}(k / \sqrt{n})],
\end{aligned}
$$

$$
E=-\hat{\Pi}_{z}(k / \sqrt{n})-\partial_{z} \hat{\Pi}_{z_{c}}(0)\left(z_{c}-z\right)+\hat{\Pi}_{z_{c}}(0)+\frac{k^{2}}{2 d n} \nabla_{k}^{2} \hat{\Pi}_{z_{c}}(0) .
$$

The error term can be written

$$
E=E_{1}+E_{2}+E_{3},
$$


where

$$
\begin{aligned}
& E_{1}=E_{1}(z, k / \sqrt{n})=\hat{\Pi}_{z_{c}}(k / \sqrt{n})-\hat{\Pi}_{z}(k / \sqrt{n})-\partial_{z} \hat{\Pi}_{z_{c}}(k / \sqrt{n})\left(z_{c}-z\right), \\
& E_{2}=E_{2}(z, k / \sqrt{n})=\left[\partial_{z} \hat{\Pi}_{z_{c}}(k / \sqrt{n})-\partial_{z} \hat{\Pi}_{z_{c}}(0)\right]\left(z_{c}-z\right)
\end{aligned}
$$

and

$$
E_{3}=E_{3}(k / \sqrt{n})=\hat{\Pi}_{z_{c}}(0)-\hat{\Pi}_{z_{c}}(k / \sqrt{n})+\frac{k^{2}}{2 d n} \nabla_{k}^{2} \hat{\Pi}_{z_{c}}(0)
$$

By (4.8),

$$
\frac{1}{\hat{F}_{z}(k / \sqrt{n})}=\frac{1}{\alpha-\beta z}-\frac{E}{(\alpha-\beta z) \hat{F}_{z}(k / \sqrt{n})} .
$$

We now insert (4.16) into (4.6). The integral corresponding to the first term on the right side of (4.16) is $\beta^{n T} \alpha^{-(n T+1)}$. A straightforward calculation using the definition of $D$ in (3.5) and the fact that $1-\hat{D}(u) \sim-u^{2} \nabla^{2} \hat{D}(0) / 2$ shows that

$$
\begin{aligned}
\frac{\beta^{n T}}{\alpha^{n T+1}} & \sim \frac{1}{-\partial_{z} \hat{F}_{z_{c}}(0) z_{c}^{n T+1}}\left[1-\frac{D k^{2}}{2 d n}\right]^{n T} \\
& \sim \frac{1}{-\partial_{z} \hat{F}_{z_{c}}(0) z_{c}^{n T+1}} \exp \left[-D k^{2} t / 2 d\right] .
\end{aligned}
$$

Comparing (4.5), the theorem follows from (4.17) if it can be shown that

$$
\frac{1}{2 \pi i} \oint \frac{E}{(\alpha-\beta z) \hat{F}_{z}(k / \sqrt{n})} \frac{d z}{z^{n T+1}}=o\left(z_{c}^{-n T}\right) .
$$

We show that (4.18) holds by using Lemma 3.3.

The first step is to obtain lower bounds on the two factors in the denominator of the integrand of (4.18). We begin with $|\alpha-\beta z|=\beta|\alpha / \beta-z|$. For large $n, \beta$ is bounded away from zero by Theorem 2.8. Also, it can be seen from (4.17) that $\alpha / \beta \geqq z_{c}$ for $n$ large. Hence there is a positive constant such that for large $n$ and $|z| \leqq z_{c}$,

$$
|\alpha-\beta z| \geqq \text { const. }\left|z_{c}-z\right| \text {. }
$$

For a lower bound on $\left|\hat{F}_{z}(k / \sqrt{n})\right|$, we write

$$
\hat{F}_{z}(k / \sqrt{n})=\hat{F}_{z}(k / \sqrt{n})-\hat{F}_{z_{c}}(k / \sqrt{n})+\hat{F}_{z_{c}}(k / \sqrt{n}) .
$$

By Corollary 3.8 and Lemma 3.2, the first two terms on the right side combine to give $-\partial_{z} \hat{F}_{z_{c}}(k / \sqrt{n})\left(z_{c}-z\right)+O\left(\left|z_{c}-z\right|^{1+\varepsilon}\right)$. By the dominated convergence theorem the derivative appearing here is continuous in $k$, and hence differs from its value at $k=0$ by $o(1)$. Thus we have

$$
\hat{F}_{z}(k / \sqrt{n})=\left[-\partial_{z} \hat{F}_{z_{c}}(0)+O\left(\left|z_{c}-z\right|^{\varepsilon}\right)+o(1)\right]\left(z_{c}-z\right)+\hat{F}_{z_{c}}(k / \sqrt{n}) .
$$

By Theorem 2.8, the last term on the right side is nonnegative. Since the first term in square brackets on the right side is also nonnegative by Theorem 2.8 , it follows that for $z$ in a small neighbourhood of $z_{c}$ (inside the closed disk of radius $z_{c}$ ), the right 
side of (4.21) is bounded below by const. $\left|z_{c}-z\right|$ (for large $n$ ). Outside of this neighbourhood, by Lemma 3.9 there is a constant $c>0$ such that $\left|\hat{F}_{z}(0)\right| \geqq c$. Hence $\left|\hat{F}_{z}(k / \sqrt{n})\right| \geqq c / 2$ if $n$ is sufficiently large, since by Theorem 2.6 there is a bound on $\left|\nabla_{k} \hat{F}_{z}(k)\right|$ which is uniform in $k$ and $|z| \leqq z_{c}$. Therefore for $|z| \leqq z_{c}$ we have

$$
\left|\hat{F}_{z}(k / \sqrt{n})\right| \geqq \text { const. }\left|z_{c}-z\right| .
$$

We now turn to upper bounds on $E_{i}$ for $i=1,2,3$. Beginning with $E_{1}$, it follows from (4.19), (4.22), and a straightforward calculation using Corollary 3.8 and Lemma 3.2 that

$$
\left|\frac{d}{d z} \frac{E_{1}}{(\alpha-\beta z) \hat{F}_{z}(k / \sqrt{n})}\right| \leqq O\left(\left|z_{c}-z\right|^{\varepsilon-2}\right),
$$

and hence by Lemma 3.3(ii) (4.18) is satisfied if $E$ is replaced by $E_{1}$.

For $E_{2}$, we show

$$
\left|E_{2}\right| \leqq n^{-\varepsilon / 2}\left|z_{c}-z\right|
$$

which suffices by Lemma 3.3(i). To do so we write $\pi_{m}(x)$ for the coefficient of $z^{m}$ in $\Pi_{z}(x)$, so that

$$
\partial_{z} \hat{\Pi}_{z_{c}}(k / \sqrt{n})-\partial_{z} \hat{\Pi}_{z_{c}}(0)=-\sum_{x, m} m \pi_{m}(x) z_{c}^{m-1}[1-\cos (k \cdot x / \sqrt{n})] .
$$

Since $|1-\cos t| \leqq O\left(t^{\varepsilon}\right)$ for small $\varepsilon \leqq 2$, and since $|x|^{\varepsilon}\left|\pi_{m}(x)\right| \leqq m^{\varepsilon}\left|\pi_{m}(x)\right|$, the right side of (4.25) is $O\left(n^{-\varepsilon / 2}\right)$ by Corollary 3.8, which gives (4.24).

Finally, for $E_{3}$ we use symmetry to write

$$
E_{3}=\sum_{x, m} \pi_{m}(x) z_{c}^{m}\left[1-\cos (k \cdot x / \sqrt{n})-\frac{(k \cdot x)^{2}}{2 n}\right] .
$$

For small positive $\varepsilon, \quad\left|1-\cos t-t^{2} / 2\right| \leqq O\left(|t|^{2+\varepsilon}\right)$. Since $\left|x^{2+\varepsilon}\right|\left|\pi_{m}(x)\right|$ $\leqq m^{\varepsilon}|x|^{2}\left|\pi_{m}(x)\right|$, it follows from Corollary 3.8 that $\left|E_{3}\right| \leqq O\left(n^{-1-\varepsilon / 2}\right)$. Then Lemma 3.3(i) gives (4.18) for $E$ replaced by $E_{3}$.

4.2. The Finite-Dimensional Distributions. In this section we complete the proof of Theorem 1.6 by showing that (4.3) holds for $N \geqq 2$. The following lemma, whose proof can be found in [34], will be used for this purpose. (See (2.5) and (2.7) for the definitions of $K[a, b]$ and $J[a, b]$.)

Lemma 4.2. For any integers $0 \leqq m \leqq b$,

$$
K[0, b]=\sum_{I \ni m} K\left[0, I_{1}\right] J\left[I_{1}, I_{2}\right] K\left[I_{2}, b\right],
$$

where the sum over $I$ is the sum over intervals $\left[I_{1}, I_{2}\right]$ of integers with either $0 \leqq I_{1}<m<I_{2} \leqq$ or $I_{1}=I_{2}=m$.

The proof of (4.3) is by induction on $N$, with the case $N=1$ having been treated in the previous section. To perform the induction step, some flexibility is needed in the number of steps in the walk. Let $g=\left(g_{n}\right)$ be any sequence satisfying $0 \leqq g_{n} \leqq n^{-1 / 2}$, and let $\mathbf{T}=\left(t_{1}, t_{2}, \ldots, t_{N-1}, T\right)$, where $T=t_{N}\left(1-g_{n}\right)$. It suffices to prove the following theorem. 
Theorem 4.3. Let $N \geqq 2$. Suppose that

$$
\lim _{n \rightarrow \infty} c_{n T}^{-1} M(\mathbf{k} / \sqrt{n}, n \mathbf{T})=\exp \left[-\frac{D}{2 d} \sum_{i=1}^{N}\left(k^{(i)}\right)^{2}\left(t_{i}-t_{i-1}\right)\right]
$$

holds uniformly in $g$, when $N$ is replaced by $N-1$. Then in fact (4.27) holds as stated, uniformly in $\mathrm{g}$.

Proof. The proof closely follows Sect. 4 of [34], and more details on the proof can be found there. To simplify the notation we write $\kappa=\left(\kappa_{1}, \ldots, \kappa_{N}\right)=\mathbf{k} / \sqrt{n}$. By (4.2), and Lemma 4.2 with $m=n t_{N-1}$,

$$
M(\kappa, n \mathbf{T})=\sum_{I \ni n t_{N-1}} \sum_{\omega:|\omega|=n T} e^{i \kappa \cdot \Delta \omega(n \mathbf{T})} K\left[0, I_{1}\right] J\left[I_{1}, I_{2}\right] K\left[I_{2}, n T\right] .
$$

In (4.28) we factor the walk $\omega$ into three independent subwalks on the subintervals $\left[0, I_{1}\right], I=\left[I_{1}, I_{2}\right]$ and $\left[I_{2}, n T\right]$. We fix a sequence $b_{n}$ with $\lim _{n \rightarrow \infty} b_{n}=\infty$ and $b_{n}=o\left(n^{1 / 2}\right)$, for example $b_{n}=n^{1 / 4}$. It will become apparent that the significant contribution to the right side of (4.28) is due to intervals $I$ with $|I| \leqq b_{n}$. We take $n$ sufficiently large that for such $I, n t_{N-2}<I_{1} \leqq n t_{N-1} \leqq I_{2}<n T$.

By factoring the exponential in the right side and summing only over those $I$ with $|I| \leqq b_{n}$, a resummation can be performed to give

$$
\begin{aligned}
M_{\mathbf{k}} \equiv & \sum_{\substack{I \ni n t_{N-1} \\
|I| \leqq b_{n}}} M\left(\kappa_{1}, \ldots, \kappa_{N-1} ; n t_{1}, \ldots, n t_{N-2}, I_{1}\right) \\
& \times \sum_{\omega:|\omega|=|I|} E_{1}(\omega, I) J[0,|I|] \hat{c}_{n T-I_{2}}\left(\kappa_{N}\right),
\end{aligned}
$$

where

$$
\begin{aligned}
E(\omega, I) & =\exp \left[i \kappa_{N-1} \cdot \omega\left(n t_{N-1}-I_{1}\right)+i \kappa_{N} \cdot \omega\left(I_{2}-n t_{N-1}\right)\right] \\
& =1+O\left(b_{n} n^{-1 / 2}\right)
\end{aligned}
$$

uniformly in $\omega$ and $|I| \leqq b_{n}$. For $|I| \leqq b_{n}$ and $n$ sufficiently large, $I_{1} \in$ $\left[n t_{N-1}\left(1-n^{-1 / 2}\right), n t_{N-1}\right]$. Hence by the induction hypothesis,

$$
\begin{aligned}
& M\left(\kappa_{1}, \ldots, \kappa_{N-1} ; n t_{1}, \ldots, n t_{N-2}, I_{1}\right) \\
& =c_{I_{1}}\left[\exp \left[-\frac{D}{2 d} \sum_{i=1}^{N-1}\left(k^{(i)}\right)^{2}\left(t_{i}-t_{i-1}\right)\right]+E_{2}(I)\right],
\end{aligned}
$$

where $\left|E_{2}(I)\right|=o(1)$ uniformly in $|I| \leqq b_{n}$. Similarly it follows from Theorem 4.1 that for $|I| \leqq b_{n}$,

$$
\hat{c}_{n T-I_{2}}\left(\kappa_{n}\right)=c_{n T-I_{2}}\left[\exp \left[-\frac{D}{2 d}\left(k^{(N)}\right)^{2}\left(t_{N}-t_{N-1}\right)\right]+E_{3}(I)\right],
$$

where $\left|E_{3}(I)\right|=o(1)$ uniformly in $|I| \leqq b_{n}$.

Substituting (4.30)-(4.32) into (4.29) leads to

$$
M_{\mathrm{k}}=\exp \left[-\frac{D}{2 d} \sum_{i=1}^{N}\left(k^{(i)}\right)^{2}\left(t_{i}-t_{i-1}\right)\right] M_{0}^{\leqq}+A,
$$


where

$$
|A| \leqq o(1) \sum_{\substack{I \ni n t_{N-1} \\|I| \leqq b_{n}}} c_{I_{1}} \sum_{|\omega|=|I|}|J[0,|I|]| c_{n T-I_{2}}
$$

Since $M(\mathbf{0}, n \mathbf{T})=c_{n T}$, we have $M_{0}^{\leqq}=c_{n T}-M_{0}^{>}$, where $M_{\mathrm{k}}^{>}$is defined as in (4.29) with the sum over $|I| \leqq b_{n}$ replaced by the sum over $|I|>b_{n}$. Hence

$$
\begin{aligned}
c_{n T}^{-1} M(\kappa, n \mathbf{T})= & \exp \left[-\frac{D}{2 d} \sum_{i=1}^{N}\left(k^{(i)}\right)^{2}\left(t_{i}-t_{i-1}\right)\right]\left[1-c_{n T}^{-1} M_{\mathbf{0}}^{>}\right] \\
& +c_{n T}^{-1} A+c_{n T}^{-1} M_{\mathbf{k}}^{>} .
\end{aligned}
$$

Now by Theorem 1.1(a) and (4.34),

$$
c_{n T}^{-1}|A| \leqq o(1) \sum_{|I|=1}^{b_{n}}|I| \sum_{|\omega|=|I|}|J[0, I]| z_{c}^{|I|}
$$

(here the sum is no longer a sum over intervals; now $|I|$ is merely a summation index). In (4.36) the factor $|I|$ counts the number of possibilities for $n t_{N-1} \in I$. Extending the summation over $|I|$ on the right side to infinity, it follows from the (absolute) bound on $\partial_{z} \hat{\Pi}$ of Theorem 2.6 that

$$
c_{n T}^{-1}|A| \leqq o(1) \text {. }
$$

It suffices now to show that $c_{n T}^{-1} M_{\mathbf{k}}^{>}=o(1)$ as $n \rightarrow \infty$. Arguing as in the previous paragraph,

$$
c_{n T}^{-1}\left|M_{\mathbf{k}} \mathbf{O}\right| \leqq O(1) \sum_{|I|=b_{n}+1}^{\infty}|I| \sum_{|\omega|=|I|}|J[0, I]| z_{c}^{|I|} .
$$

The right side goes to zero as $n \rightarrow \infty$, since by Theorem 2.6

$$
\sum_{|I|=1}^{\infty}|I| \sum_{|\omega|=|I|}|J[0,|I|]| z_{c}^{|I|}<\infty .
$$

\section{The Infinite Self-Avoiding Walk}

In this section we give the proof of Theorem 1.7. The proof follows the basic approach used in [24] to prove existence of the infinite self-avoiding walk for $d$ sufficiently large, but is simpler since use of a finite memory is avoided.

The infinite self-avoiding walk was defined in [23] as follows. Given $n \geqq m$ and an $m$-step self-avoiding walk $\omega$, we let $P_{m, n}(\omega)$ denote the fraction of $n$-step walks whose first $m$ steps are given by $\omega$, i.e. $P_{m, n}(\omega)$ is the fraction of $n$-step self-avoiding walks which extend $\omega$. Then we define

$$
P_{m}(\omega)=\lim _{n \rightarrow \infty} P_{m, n}(\omega)
$$

if the limit exists. If the limit does exist, then the probability measures $P_{m}$ on $m$-step walks will be consistent in the sense that for each $n \geqq m$ and each $m$-step selfavoiding walk $\omega$,

$$
P_{m}(\omega)=\sum_{\eta>\omega} P_{n}(\eta)
$$


where the sum is over all $n$-step self-avoiding walks $\eta$ which extend $\omega$. This consistency property allows for the definition via cylinder sets of a measure $P_{\infty}$ on the set of all infinite self-avoiding walks (see [24] for more details). The measure $P_{\infty}$ is the infinite self-avoiding walk.

The closest results to existence of the limit (5.1) in general dimensions $d \geqq 2$ are the proofs in [25] that for any $m$-step self-avoiding walk $\omega$ which can be extended to an infinite self-avoiding walk, $\liminf _{n \rightarrow \infty} P_{m, n}(\omega)>0$ and $\lim _{n \rightarrow \infty} P_{m, n+2}(\omega) / P_{m, n}(\omega)=1$. The remainder of this section is devoted to a proof that the limit in (5.1) exists for $d \geqq 5$.

Given a nonnegative integer $m$, let $\mathbf{k}=\left(k^{(1)}, \ldots, k^{(m)}\right)$, where $k^{(i)} \in[-\pi, \pi]^{d}$. Given $n \geqq m$ and an $n$-step self-avoiding walk $\omega$, let $\omega_{m}$ be the first $m$ steps of $\omega$, and

$$
\mathbf{k} \cdot \omega_{m}=\sum_{i=1}^{m} k^{(i)} \cdot \omega(i)
$$

Let

$$
\bar{\varphi}_{m, n}(\mathbf{k})=\sum_{|\omega|=n: \text { simple }} e^{i \mathbf{k} \cdot \omega_{m}} K[0, n],
$$

where the sum is over all $n$-step simple random walks and $K$ was introduced in (2.5), and let

$$
\varphi_{m, n}(\mathbf{k})=\sum_{|\omega|=m: \text { saw }} e^{i \mathbf{k} \cdot \omega} P_{m, n}(\omega)=\frac{1}{c_{n}} \bar{\varphi}_{m, n}(\mathbf{k}) .
$$

Since $\left\{P_{m, n}\right\}_{n}$ is clearly tight, a standard convergence theorem (see [4], p. 46) implies that existence of the limit (5.1) follows from existence of the limit

$$
\varphi_{m}(\mathbf{k})=\lim _{n \rightarrow \infty} \varphi_{m, n}(\mathbf{k})
$$

for $\mathbf{k} \in[-\pi, \pi]^{m d}$.

For $m \geqq 0$ we define a quantity similar to the two-point function $\hat{G}_{z}(k)$ by

$$
\Gamma_{z}(\mathbf{k}, m)=\sum_{n=m}^{\infty} \bar{\varphi}_{m, n}(\mathbf{k}) z^{n}
$$

Since $\left|\Gamma_{z}(\mathbf{k}, m)\right| \leqq \chi(|z|)$, this power series converges for $|z|<z_{c}$. We define a quantity similar to $\hat{\Pi}_{z}(k)$, again for $m \geqq 0$, by

$$
\Psi_{z}(\mathbf{k}, m)=\sum_{s=m}^{\infty} z^{s} \sum_{|\omega|=s: \text { simple }} e^{i \mathbf{k} \cdot \omega_{m}} J[0, s] .
$$

It follows from the absolute bound on the lace expansion of Theorem 2.6 that for $v=0,1, \partial_{z}^{v} \Psi_{z}(\mathbf{k}, m)$ is bounded by a finite constant uniformly in $\mathbf{k}$ and $|z| \leqq z_{c}$. For $j<m$ we define $\overline{\mathbf{k}}_{j}=\left(k^{(j+1)}, \ldots, k^{(m)}\right)$. The following identity is proved in [24] along the lines of the proof in [8] of Theorem 2.2.

Lemma 5.1. For $m \geqq 1$ and for any $z$ for which both sides make sense,

$$
\begin{aligned}
\Gamma_{z}(\mathbf{k}, m)= & 2 d z \hat{D}\left(\sum_{j=1}^{m} k^{(j)}\right) \Gamma_{z}\left(\overline{\mathbf{k}}_{1}, m-1\right) \\
& +\sum_{s=2}^{m-1} z^{s} \sum_{|\omega|=s} \exp \left(i \sum_{j=1}^{m} k^{(j)} \cdot \omega(\min \{j, s\})\right) J[0, s] \Gamma_{z}\left(\overline{\mathbf{k}}_{s}, m-s\right) \\
& +\Psi_{z}(\mathbf{k}, m) \chi(z) .
\end{aligned}
$$


Let $N_{z}(\mathbf{k}, m)=\chi(z)^{-1} \Gamma_{z}(\mathbf{k}, m)$. Lemma 5.1 and induction on $m$ then can be used to argue that for $v=0,1, \partial_{z}^{v} N_{z}(\mathbf{k}, m)$ is uniformly bounded in $\mathbf{k}$ and $|z| \leqq z_{c}$.

To prove existence of the limit (5.5), we proceed as follows. By definition of $N_{z}$,

$$
\begin{aligned}
\bar{\varphi}_{m, n}(\mathbf{k}) & =\frac{1}{2 \pi i} \oint N_{z}(\mathbf{k}, m) \chi(z) \frac{d z}{z^{n+1}} \\
& =N_{z_{c}}(\mathbf{k}, m) c_{n}+\frac{1}{2 \pi i} \oint\left[N_{z}(\mathbf{k}, m)-N_{z_{c}}(\mathbf{k}, m)\right] \chi(z) \frac{d z}{z^{n+1}},
\end{aligned}
$$

where the contour is a small circle centred at the origin. It suffices to show that the second term on the right side is $o\left(c_{n}\right)$, which by Theorem 1.1(a) is equivalent to $o\left(z_{c}^{-n}\right)$. Hence by Lemma 3.3(ii) it suffices to show that for $|z| \leqq z_{c}$,

$$
\left|\frac{d}{d z}\left[N_{z}(\mathbf{k}, m)-N_{z_{\mathbf{c}}}(\mathbf{k}, m)\right] \chi(z)\right| \leqq O\left(\left|z_{c}-z\right|^{-1}\right) .
$$

Now since $\left|\partial_{z} N_{z}\right|$ is uniformly bounded for $|z| \leqq z_{c}$,

$$
\frac{d}{d z}\left[N_{z}(\mathbf{k}, m)-N_{z_{c}}(\mathbf{k}, m)\right] \chi(z)=O(1) \chi(z)+O\left(\left|z_{c}-z\right|\right) \frac{d}{d z} \chi(z) .
$$

The first term on the right side is $O\left(\left|z_{c}-z\right|^{-1}\right)$ by Lemma 3.9. It follows easily from Theorem 2.6 and Lemma 3.9 that the second term on the right side is also $O\left(\left|z_{c}-z\right|^{-1}\right)$. Thus we have (5.9) and hence by Lemma 3.3(ii) the second term on the right side of (5.8) is $O\left(z_{c}^{-n} n^{-\alpha}\right)$, for every $\alpha<1$. This completes the proof of Theorem 1.7 .

Acknowledgements. The authors have enjoyed and benefitted from conversations with David Brydges, Alan Sokal and Michael Aizenman.

\section{References}

1. Aizenman, M.: Geometric analysis of $\varphi^{4}$ fields and Ising models, Parts I and II. Commun. Math. Phys. 86, 1-48 (1982)

2. Aragão de Carvalho, C., Caracciolo, S., Fröhlich, J.: Polymers and $g|\phi|^{4}$ theory in four dimensions. Nucl. Phys. B 215 [FS7], 209-248 (1983)

3. Arnaudon, D., Iagolnitzer, D., Magnen, J.: Weakly self-avoiding polymers in four dimensions. Rigorous results. Phys. Lett. B 273, 268-272 (1991)

4. Billingsley, P.: Convergence of probability measures. New York, Chichester, Brisbane, Toronto: John Wiley and Sons 1968

5. Bovier, A., Felder, G., Fröhlich, J.: On the critical properties of the Edwards and the self-avoiding walk model of polymer chains. Nucl. Phys. B 230 [FS10], 119-147 (1984)

6. Brydges, D.C.: A short course on cluster expansions. In Osterwalder, K., Stora, R. (eds.), Critical Phenomena, Random Systems, Gauge Theories. Amsterdam, New York, Oxford, Tokyo: North-Holland (Les Houches 1984) 1986

7. Brydges, D.C., Evans, S.N., Imbrie, J.: Self-avoiding walk on a hierarchical lattice in four dimensions. Ann. Probab. 20, 82-124 (1992)

8. Brydges, D.C., Spencer, T.: Self-avoiding walk in 5 or more dimensions. Commun. Math. Phys. 97, 125-148 (1985)

9. Chayes, J.T., Chayes, L.: Ornstein-Zernike behavior for self-avoiding walks at all noncritical temperatures. Commun. Math. Phys. 105, 221-238 (1986) 
10. Fröhlich, J.: On the triviality of $\varphi_{d}^{4}$ theories and the approach to the critical point in $d \geqq 4$ dimensions. Nucl. Phys. B200 [FS4], 281-296 (1982)

11. Guttmann, A.J.: Bounds on connective constants for self-avoiding walks. J. Phys. A: Math. Gen. 16, 2233-2238 (1983)

12. Guttmann, A.J.: On the critical behaviour of self-avoiding walks: II. J. Phys. A: Math. Gen. 22, 2807-2813 (1989)

13. Hammersley, J.M.: Percolation processes, II. Connective constants. Proc. Camb. Phil. Soc. 53, 642-645 (1957)

14. Hammersley, J.M., Welsh, D.J.A.: Further results on the rate of convergence to the connective constant of the hypercubical lattice. Quart. J. Math. Oxford 13, (2), 108-110 (1962)

15. Hara, T.: Mean field critical behaviour for correlation length for percolation in high dimensions. Prob. Th. Rel. Fields 86, 337-385 (1990)

16. Hara, T., Slade, G.: Mean-field critical behaviour for percolation in high dimensions. Commun. Math. Phys. 128, 333-391 (1990)

17. Hara, T., Slade, G.: On the upper critical dimension of lattice trees and lattice animals. J. Stat. Phys. 59, 1469-1510 (1990)

18. Hara, T., Slade, G.: Critical behaviour of self-avoiding walk in five or more dimensions. Bull. A.M.S. 25, 417-423 (1991)

19. Hara, T., Slade, G.: The number and size of branched polymers in high dimensions. J. Stat. Phys. (In press)

20. Hara, T., Slade, G.: The lace expansion for self-avoiding walk in five or more dimensions. Rev. Math. Phys. (In press)

21. Kesten, H.: On the number of self-avoiding walks. J. Math. Phys. 4, 960-969 (1963)

22. Kesten, H.: On the number of self-avoiding walks. II. J. Math. Phys. 5, 1128-1137 (1964)

23. Lawler, G.: A self-avoiding random walk. Duke Math. J. 47, 655-693 (1980)

24. Lawler, G.: The infinite self-avoiding walk in high dimensions. Ann. Probab. 17, 1367-1376 (1989)

25. Madras, N.: End patterns of self-avoiding walks. J. Stat. Phys. 53, 689-701 (1988)

26. Madras, N.: Bounds on the critical exponent of self-avoiding polygons. In: Durrett, R., Kesten, H. (eds.), Random walks, Brownian motion and interacting particle systems. Boston: Birkhäuser 1991

27. Madras, N., Slade, G.: The self-avoiding walk. In preparation. To appear in the Birkhäuser series Probability and Its Applications

28. Madras, N., Sokal, A.D.: The pivot algorithm: A highly efficient Monte Carlo method for the self-avoiding walk. J. Stat. Phys. 50, 109-186 (1988)

29. Nguyen, B.G., Yang, W-S.: Triangle condition for oriented percolation in high dimensions. Preprint (1991)

30. Nienhuis, B.: Exact critical exponents of the $O(n)$ models in two dimensions. Phys. Rev. Lett. 49, 1062-1065 (1982)

31. Nienhuis, B.: Critical behaviour of two-dimensional spin models and charge asymmetry in the Coulomb gas. J. Stat. Phys. 34, 731-761 (1984)

32. Slade, G.: The diffusion of self-avoiding random walk in high dimensions. Commun. Math. Phys. 110, 661-683 (1987)

33. Slade, G.: Convergence of self-avoiding random walk to Brownian motion in high dimensions. J. Phys. A: Math. Gen. 21, L417-L420 (1988)

34. Slade, G.: The scaling limit of self-avoiding random walk in high dimensions. Ann. Probab. 17, 91-107 (1989)

35. Slade, G.: The lace expansion and the upper critical dimension for percolation. Lectures Appl. Math. 27, 53-63 (1991). Mathematics of Random Media, Kohler, W.E., White, B.S. (eds.) Providence, RI: A.M.S. 